\title{
Crime Scene Prediction by Detecting Threatening Objects Using Convolutional Neural Network
}

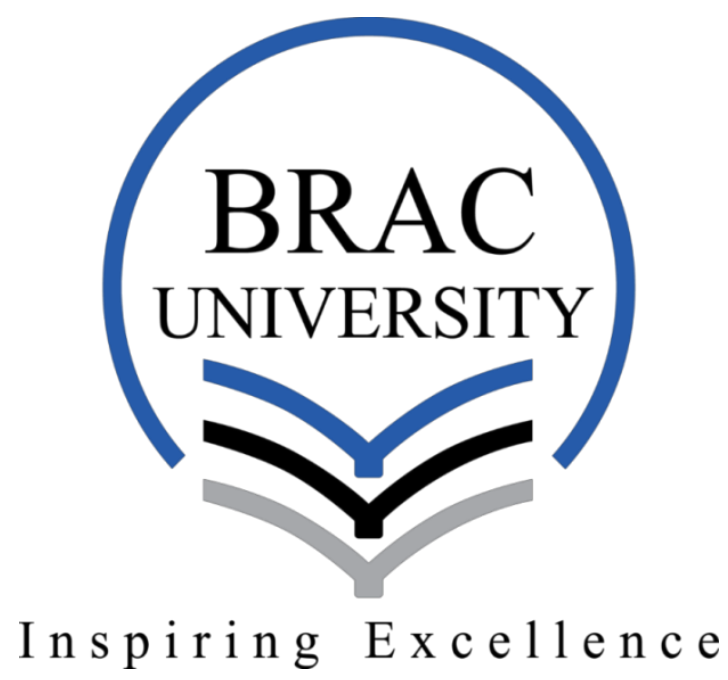

\begin{tabular}{lr} 
Supervisor: & Dr. Jia Uddin \\
\hline Mohammad Nakib & 13301044 \\
Rozin Tanvir Khan & 13101117 \\
Md. Sakibul Hasan & 13101145
\end{tabular}

Department of Computer Science and Engineering, BRAC University

Submitted on: 13 $^{\text {th }}$ April 2017 


\section{DECLARATION}

We, hereby declare that this thesis is based on the results found by ourselves. Materials ofwork found by other researcher are mentioned by reference. This Thesis, neither in whole or in part, has been previously submitted for any degree.

Signature of Supervisor

Dr. Jia Uddin
Signature of Author

Mohammad Nakib

Rozin Tanvir Khan

Md. Sakibul Hasan 


\section{ACKNOWLEDGEMENTS}

All thanks to Almighty ALLAH, the creator and the owner of this universe, the most merciful, beneficent and the most gracious, who provided us guidance, strength and abilities to complete the thesis.

We are especially thankful to Dr. Jia Uddin, our thesis supervisor, for his help, guidance and support in completion of my project. We also are thankful to the BRAC University Faculty Staffs of the Computer Science \& Engineering, who have been a light of guidance for us in the whole study period at BRAC University, particularly in building our base in education and enhancing our knowledge.

Finally, we would like to express our sincere gratefulness to our beloved parents, brothers and sisters for their love, care and support. We are grateful to all of our friends who helped us directly or indirectly to complete our thesis. 


\section{CONTENTS}

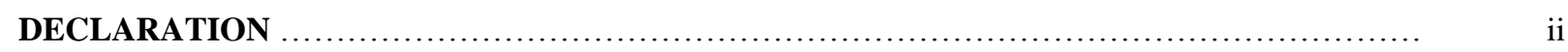

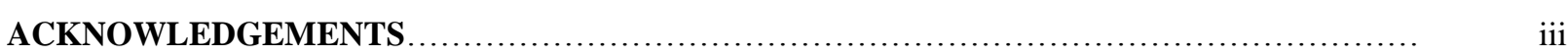

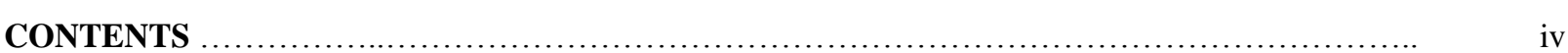

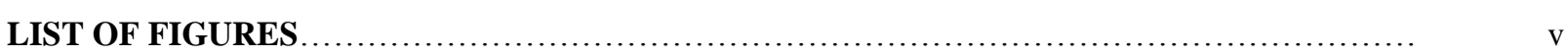

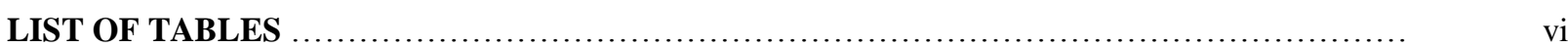

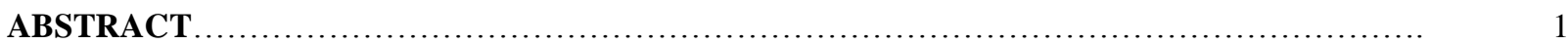

\section{CHAPTER 01: INTRODUCTION}

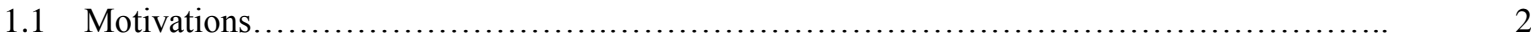

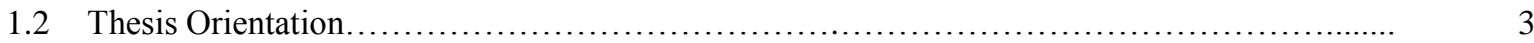

\section{CHAPTER 02: BACKGROUND INFORMATION}

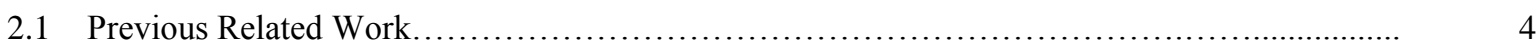

2.2 Different Models.................................................................................. 5

CHAPTER 03: PROPOSED MODEL OF CONVOLUTIONAL NEURAL NETWORK

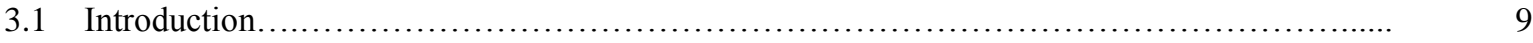

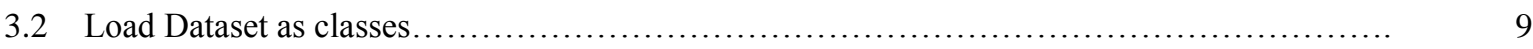

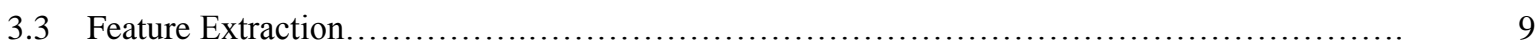

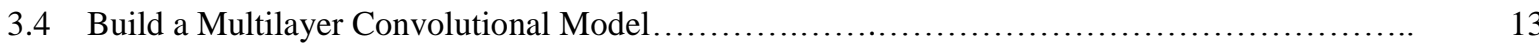

\section{CHAPTER 04: EXPERIMENTAL SETUP AND RESULT ANALYSIS}

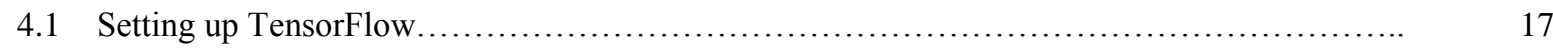

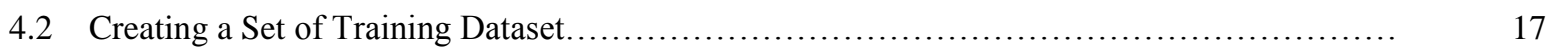

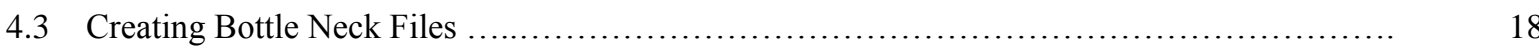

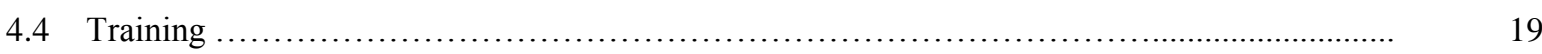

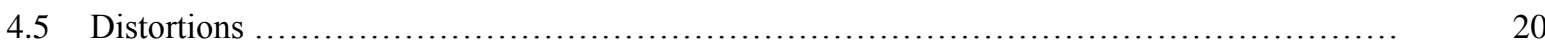

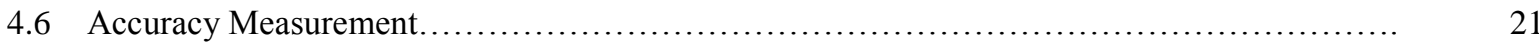

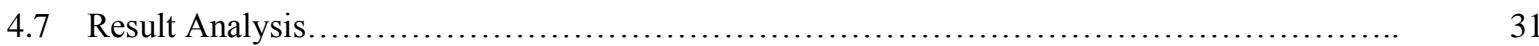

\section{CHAPTER 05: CONCLUSIONS AND FUTURE WORKS}

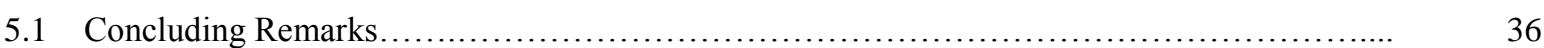

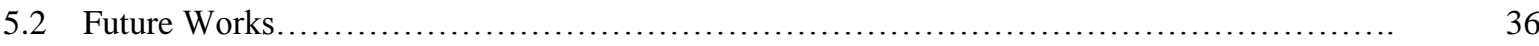




\section{LIST OF FIGURES}

Fig 2.1: Diagram of Alexnet....................................................... 5

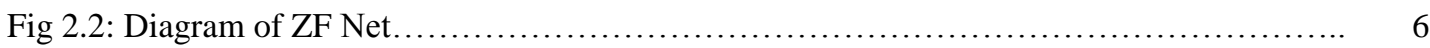

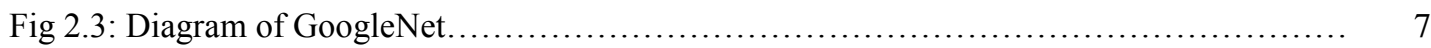

Fig 2.4: Diagram of Fully Inception Module.......................................... 8

Fig 3.1: Block Diagram of Model..................................................................................... 10

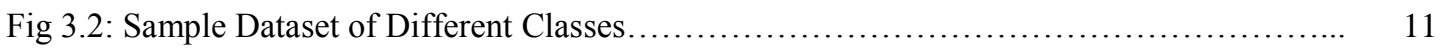

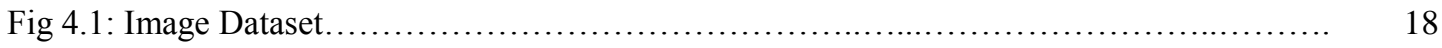

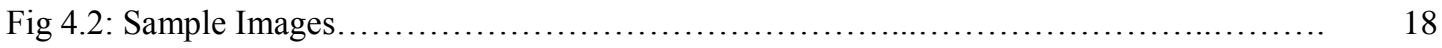

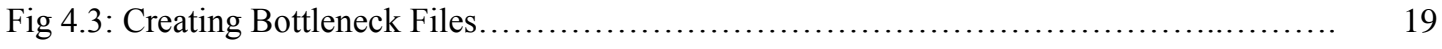

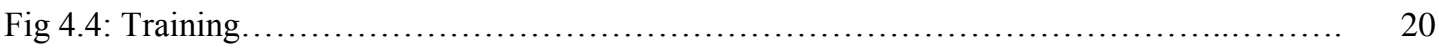

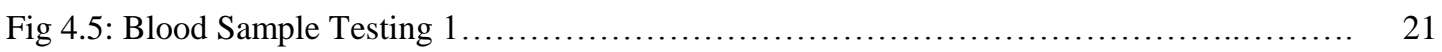

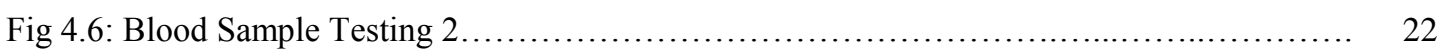

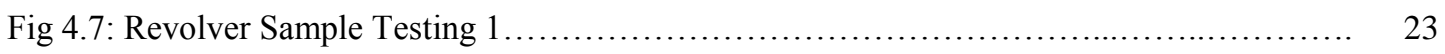

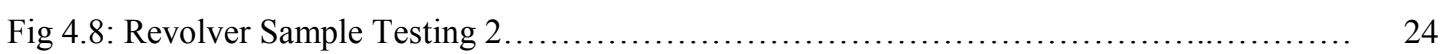

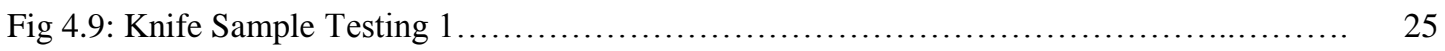

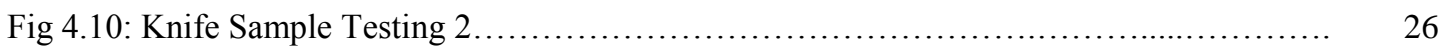

Fig 4.11: Machine Gun Sample Testing 1............................................ 27

Fig 4.12: Machine Gun Sample Testing 2 ........................................ 28

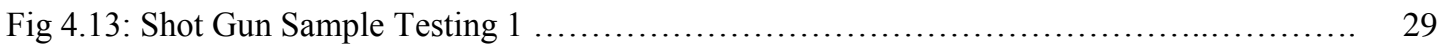

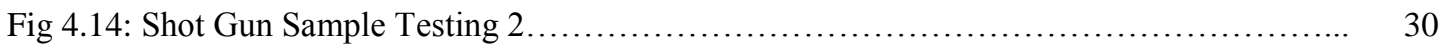

Fig 4.15: Blood Accuracy Graph................................................ 31

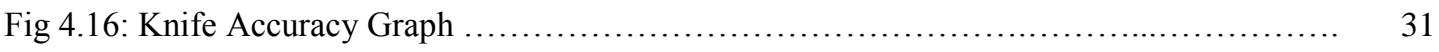

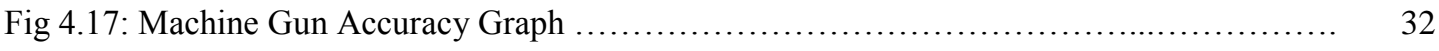

Fig 4.18: Revolver Accuracy Graph........................................... 33

Fig 4.19: Final Accuracy Graph................................................. 34 


\section{LIST OF TABLES}

Table 1 - Dataset Information.. 


\begin{abstract}
Crime scene prediction without human intervention can have outstanding impact on computer vision. In this paper, we present $\mathrm{CNN}$ in the use of detect knife, blood and gun in order to reach a prediction whether a crime has occurred in a particular image. We emphasized on the accuracy of detection so that it hardly gives us wrong alert to ensure efficient use of the system. This paper use Non linearity ReLu, Convolutional Neural Layer, Fully connected layer and dropout function of CNN to reach a result for the detection. We use Tensorflow open source platform to implement CNN to achieve our expected output. This system can achieve the test accuracy of $90.2 \%$ for the datasets we have that is very much competitive with other systems for this particular task.
\end{abstract}




\section{CHAPTER 01}

\section{INTRODUCTION}

\subsection{Motivations}

Crime scene prediction from a camera is very important while working on a field of computer vision. In modern era of science and technology, people setup surveillance cameras in different areas to get rid of crime. Still, it cannot help people as quick as people want to respond. Usually after occurring a crime scene, law enforcement agencies come to the area and take the footage from the video that was recorded at the time of crime scene. Then, law enforcement agencies analyze the video and take necessary evidence of crime scene. We believe this is very slow process to act on a crime scene. For this reason, we wanted to make a system that can quickly act on a crime scene. Moreover, there are a lot cameras being installed in different areas by law enforcement agencies or by any organization. They have to monitor all the cameras at a time with human being. If a computer system can detect the threatening objects and give alert to the authority just after detection, the proper authority can quickly take action to stop the potential criminal before he commits any crime. For example, $1^{\text {st }}$ July of 2016, an incident occurred in Dhaka in a restaurant. Terrorist went to the restaurant with guns, hand grenades and knife etc. But initially law enforcement agencies did not understand how much dangerous the terrorists are. If the camera installed over there can give information to law enforcement agencies by any media (IP camera or control from police station etc.) just after exposing the weapons, law enforcement agencies can respond to the scene very quickly and may save important lives. This incident helped us to think more deeply to make a system that can be learned to detect threatening objects. In our paper, we worked on detecting revolver, machine gun, shot gun, blood and knife using convolutional neural network.

\subsection{Thesis Orientation}

The rest of the thesis is organized as follows:

$>$ Chapter 02 includes the necessary background information regarding the proposed model of Convolutional Neural Network

$>$ Chapter 03 presents the proposed model of Convolutional Neural Network. 
$>$ Chapter 04 demonstrates the experimental setup, results and accuracy analysis.

$>$ Chapter 05 concludes the thesis and states the future research directions. 


\section{CHAPTER 02}

\section{Literature Review}

\subsection{Previous Related Work}

We emphasized on neural network instead of hand crafted features like HOG [14] or SIFT [13] to implement this. As in [15], they avoided SIFT as they points out that it is time consuming and complex. The probability of getting better results gets higher if we use neural network. In [8], Multi scale convolutional networks was used in traffic sign classification in which we found this paper can able to modify ConvNet architecture by feeding 1st stage features with 2nd stage features to the classifier. This approach helped them to get result of $98.97 \%$ accuracy.

As deep neural network (DNN) can give outstanding performance on image classification, this paper [11] focused mainly on the problems of object localization in the image. Though in our work we avoided to determine to localize of the objects rather than give more importance on determining object. In [11], their work on image classification they used the simple formulation of detection as DNN base object mask regression give strong results using multiscale course to fine procedure. It [11] can detect objects with localization but they emphasized less on detection of any particular objects like weapon.

Furthermore, in [16], they work on signal processing techniques of concealed weapon detection using neural network. It [16] shows multimeter wave radar work smoothly on the detection of concealed weapon. Again, in [9], they conclude with very important findings that help our work. They conclude that (1) automatic feature representation gives better performance than manual feature, (2) Unsupervised approach can give very close results to supervised approach and (3) Multiple instance learning outweighs the supervised learning performance.

A much-related work of our work of firearm and knife detection is [17]. It [17] proposes an algorithm that can detect firearm and knife in image. It uses OpenCV to implement this. It shows that a near zero false alarm it can generate but in order to do that it ignores positive alarms with the cost of sensitivity. So, actual percentage of detecting firearm and knife gets reduced (sensitivity 35\%). But in real life situation, even a single is missed in detecting firearm or knife, it is possible that we may fail to save many important lives. Therefore, our 
paper focused mainly on accuracy very seriously that it can get better result as much as possible unlike [17]

In this work, we use convolutional neural network to implement this. Because a simple neural network cannot give desired result we want. Therefore, we have to use convolutional neural network to get better result. In order to modify, we use ReLU, dropout and changed the input volume type in order to fit for convolutional layer.

\subsection{Different Models}

\section{AlexNet:}

AlexNet was the first model build upon using deep convolutional network. Since then, it has established the benchmark for the new upcoming neural network classifiers. The paper in which this model published on is "ImageNet Classification with Deep Convolutional Networks". This highly trained model was established by Alex Krizhevsky, Ilya Sutskever, and Geoffrey Hinton which have won 2012 ImageNet Large-Scale Visual Recognition Challenge.In the building of AlexNet a simple five layers of convolutional network )max pooling layers, dropout layers and three fully connected layers( is used alongside with ReLU for the nonlinearity functions. Through this, AlexNet was able to achieve a top 5 test error rate of $15.4 \%$. In Fig. 2.1 the diagram of this model is shown.

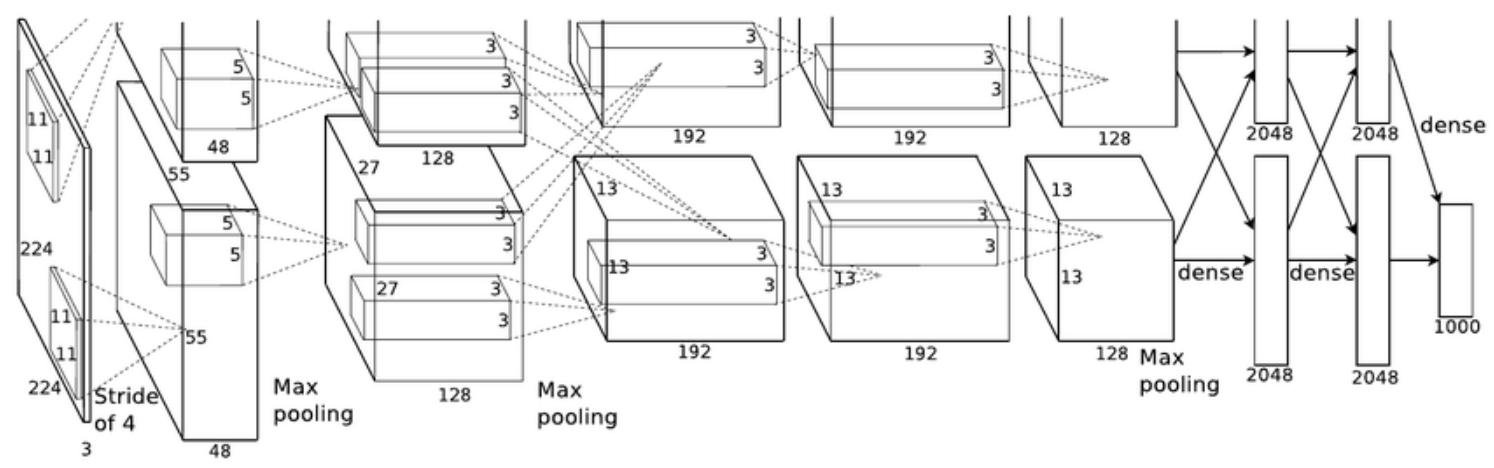

Fig 2.1: Diagram of AlexNet 


\section{ZF Net:}

In the Year 2013 in ILSVRC a large number of CNN based modules are submitted after the remarkable achievement of ALexNet in 2012. The winner of the competition on 2013 was a network built by Matthew Zeiler and Rob Fergus from NYU. Named ZF Net, this model achieved an $11.2 \%$ error rate. This model's architecture is pretty much similar to AlexNet though minor modification as instead of using $11 \times 11$ sized filters in the first layer of AlexNet, Zf Net used 7x7 filter size with decrease stride value. Also, AlexNet was trained with 15 million images, but ZF Net trained with 1.3 million images. Used ReLUs for their activation functions, cross-entropy loss for the error function, and trained using batch stochastic gradient descent. In Fig. 2.1 the diagram of this model is shown.

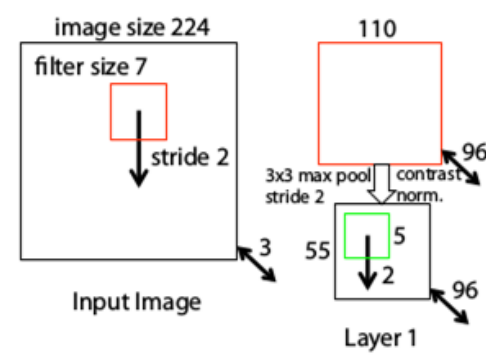

ZF Net Architecture
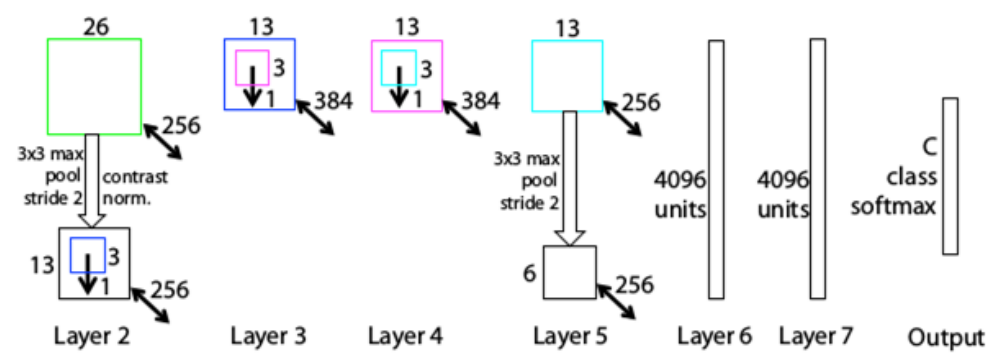

Fig 2.2: Diagram of ZF Net

\section{DeConvNet:}

The main idea behind how DeConvNet model works is that at every layer of the trained $\mathrm{CNN}$, an attached "deconvnet" which has a path back to the image pixels. An input image is fed into the CNN and activations are computed at each level. This is called forward passand the reasoning behind this whole process is that we want to examine what type of structures excite a given feature map. Let's look at the visualizations of the first and second layers. This deconvnet has the same filters as the original CNN. The inputs given here are goes through a series of reverse maxpooling, rectify, and filter operations for each preceding layer until the input space is reached.

The visualization terms and approaches which described in the ZF Net helps not only to explain the inner workings of CNNs, but also provides insight for improvements to network architectures. 


\section{VGG NET (2014):}

VGG Net Model was created based on simplicity and depth. This Model was created in 2014 best utilized with its $\mathbf{7 . 3 \%}$ error rate. Karen Simonyan and Andrew Zisserman of the University of Oxford created a 19 layer CNN that strictly used 3x3 filters with stride and pad of 1 , along with $2 \times 2$ maxpooling layers with stride 2 . The combination of two $3 \times 3$ convolutional layers has an effective receptive field of $5 \times 5$. This is the benefit of keeping smaller filter size while generating a large filter in the simulation. Another benefit is a decrease in the number of parameters. Also, with two conv layers it's enable to use two ReLU layers instead of one. Interesting to notice that the number of filters doubles after each maxpool layer. This reinforces the idea of shrinking spatial dimensions, but growing depth. This process, worked well on both image classification and localization tasks. It's used scale jittering which is a one data augmentation technique at the time of training.

\section{GoogLeNet (2015):}

GoogLeNet is a 22 layer CNN and was the winner of ILSVRC 2014 with a top 5 error rate of 6.7\%. This was one of the first CNN architectures that really strayed from the general approach of simply stacking convolutional and pooling layers on top of each other in a sequential structure. This model is also emphasized on the notable consideration of memory and power usage. In Fig. 2.3 the diagram of this model is shown.

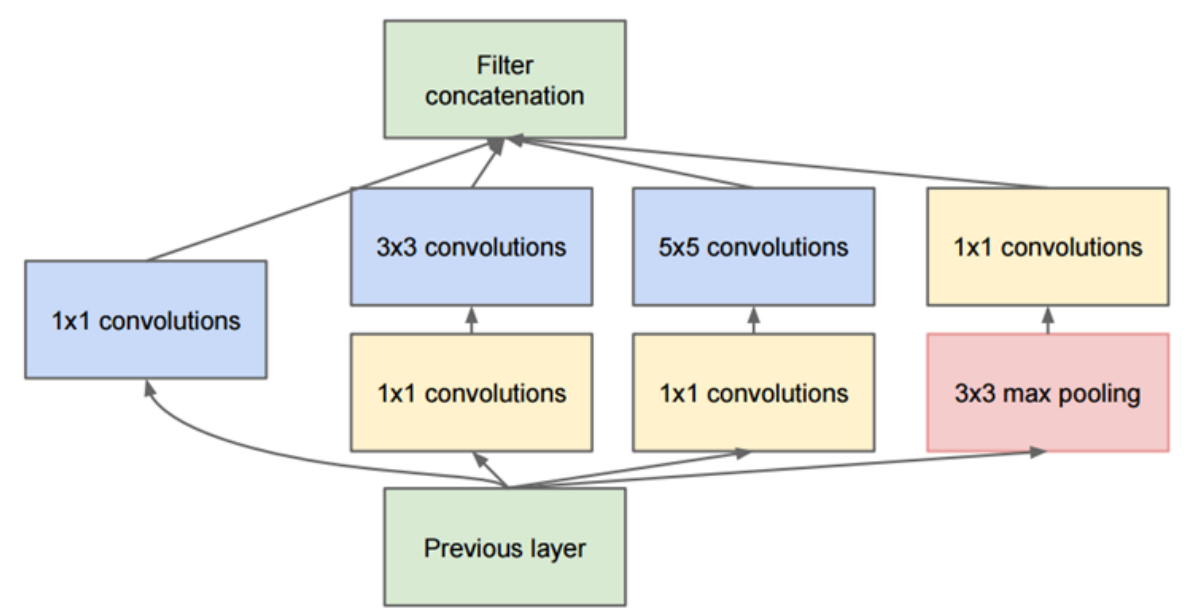

Fig 2.3: Diagram of GoogleNet 


\section{Fully Inception Module:}

In the inception module, not only the layers are implemented in sequentially but also most of the layers are working on parallel in the network. In fully inception module a total 9 Inception modules in the whole architecture, with over 100 layers are used. It uses 12x fewer parameters than big AlexNet. Utilized concepts from R-CNN for the detection model. The update version of Inception module is now established (Version 6 and 7). GoogLeNet was one of the first models that introduced the idea of parallax computing of CNN layers rather than used in stacked up sequential layers. Coming up with the Inception module, the authors showed that a creative structuring of layers can lead to improved performance and computationally efficiency. In Fig. 2.4 the diagram of thismodel is shown.

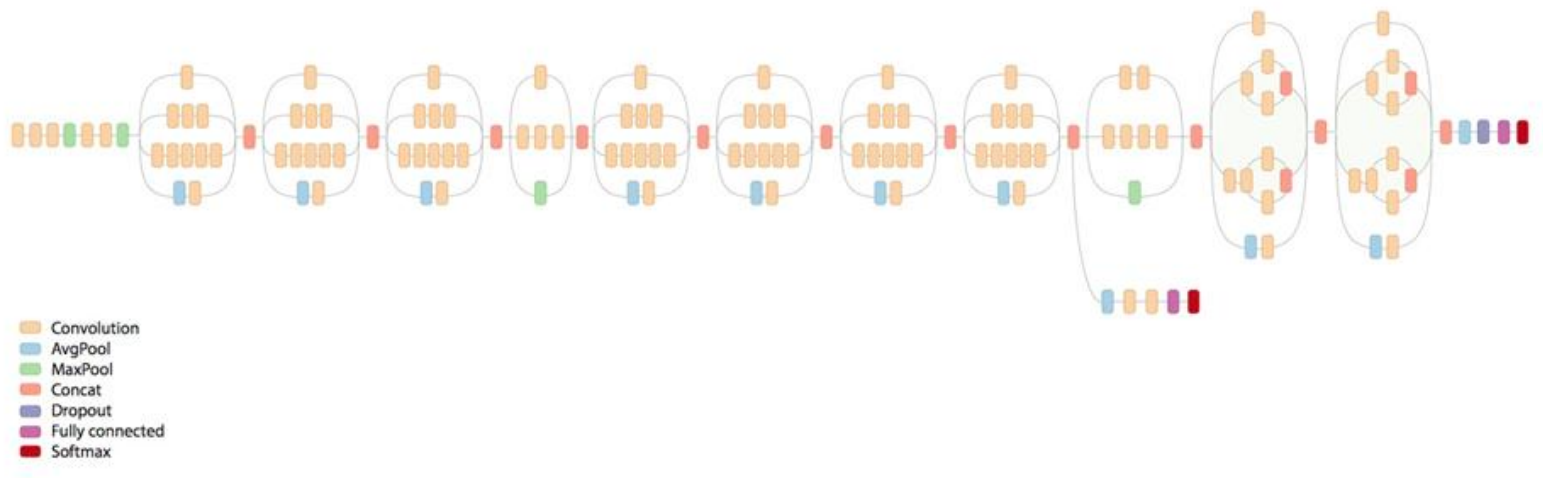

Fig 2.4: Diagram of Fully Inception Module 


\section{CHAPTER 03}

\section{PROPOSED CONVOULUTIONAL NEURAL NETWORK MODEL}

\subsection{Introduction}

As firearms, knife and blood detection from an image is our objective, we propose a simple block diagram using $\mathrm{CNN}$ which focused on ensuring accuracy rate as high as possible. In Tersorflow platform this model can identify almost all the input data with high accuracy. A block diagram the model is given on Fig 3.1.

\subsection{Load Dataset as Classes}

We want to detect knife, gun (short gun, revolver and machine gun) and blood. We make dataset which has 5 classes as input images. Every detection object has to have a single class of image in the dataset. We make the datasets using the image of 150x100 pixels (dimension). We call multiple images as batch. In this paper, batches of images are taken to train the system and shows output for particular input. Our system not only detects the objects it also shows the graph. Starting an interactive session can help to structure our system. It also helps to call computational graph to check how the system is working. Then the images are sent to the next step for the feature extraction. In Fig 3.2, the sample dataset is given according to classes.

\subsection{Feature Extraction}

To extract features from the images, we use some steps to generalize the images and turn the images into certain pattern. The main two steps of the feature extraction are "build software regression model" and "Training the model". These two steps include some sub steps.

\subsubsection{Build a Softmax Regression Model}

Initially we make a softmax regression model by making a single linear layer. But we change this single linear layer to multilayer convolutional layers.

To extract the feature from input images, we need to take some variables for computations. 

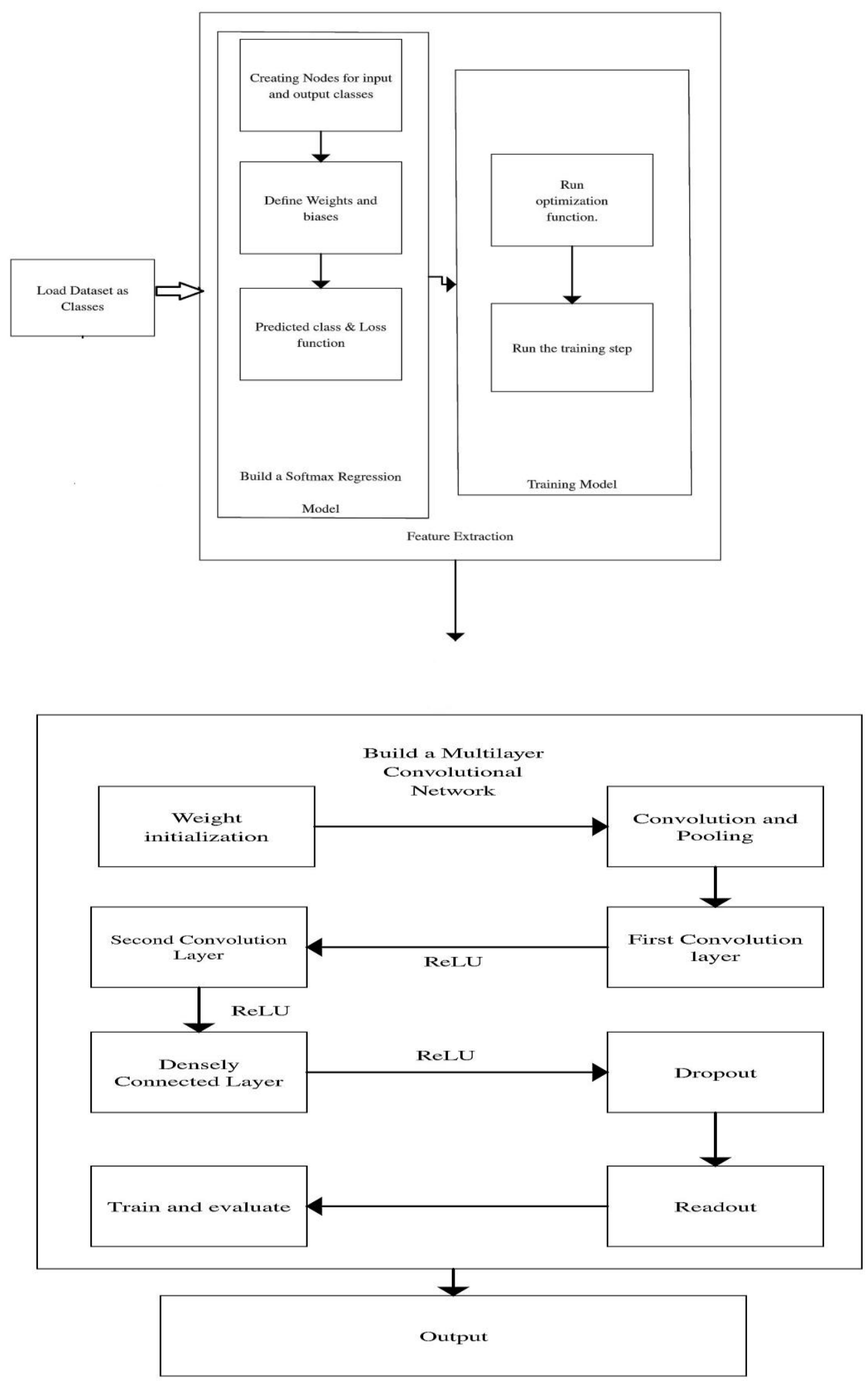

Fig 3.1: Block Diagram of Proposed Model

As mentioned above that our images which has $150 \times 100$ pixels (dimension). At first, we turn the whole image into a single linear array and our first batch of images contains 50 
images. Therefore, we make a placeholder, $X$ which has size 1500 columns (as $150 \times 100=$ 15000) and 50 rows (as 50 images for first batch). Every row contains the values of a single image.
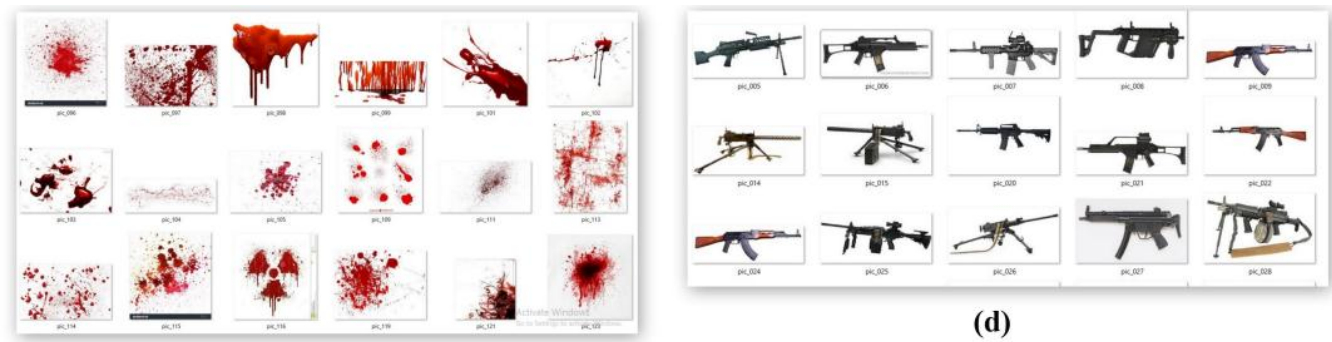

(d)

(a)

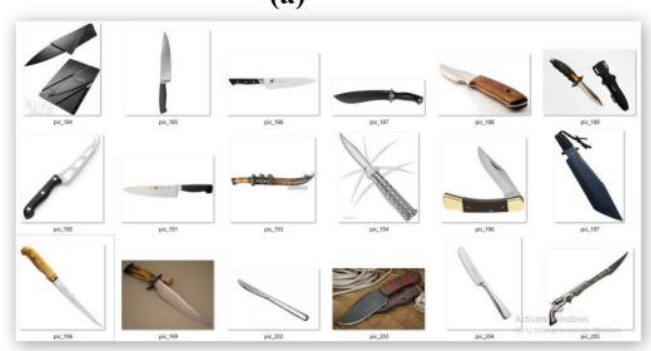

(b)

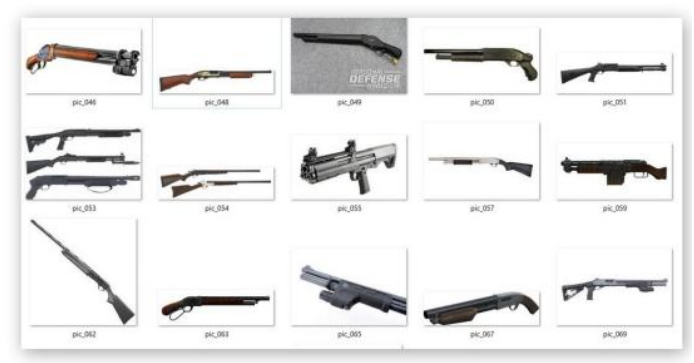

(e)

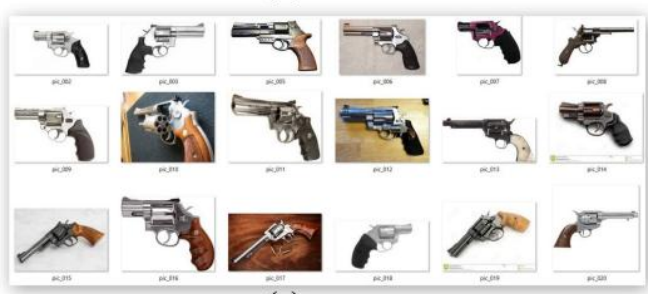

(c)

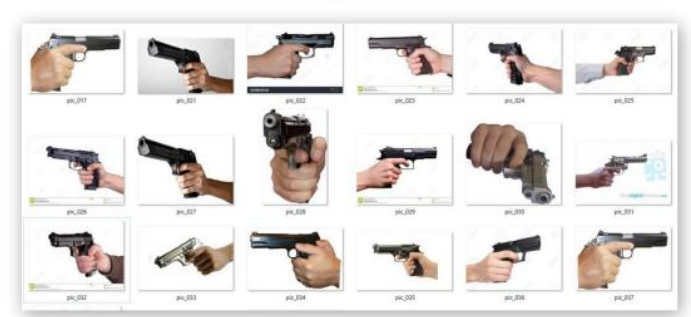

(f)

Fig. 3.2 Sample dataset of different classes (a) Blood (b) Knife (c) Revolver (d) Machine Gun (e) Shot Gun (f) Gun in hand

We also make a placeholder which contains the information of classes. The size of this matrix is $50 \times 6$. As we have 5 classes for 50 images first. The number of images in a batch changes in training step and it increases by 50 images.

We have to define our weights $\mathrm{W}$ and biases $\mathrm{b}$. For our input, the size of weights matrix is $1500 \times 6$. As the dimension of our images in datasets is $150 \times 100$ pixel (dimension) and the class number is 6 . Moreover, the size of bias matrix $b$ is $1 \times 6$ because it only contains the biases of 6 classes only for our input.

After creating placeholders and variables we now can execute the regression model. It is the multiplications of the vectorized input images $\mathrm{X}$ with weights $\mathrm{W}$ and add the bias $\mathrm{b}$ with the multiplication matrix. 
$Y=X \cdot W+b$

Here, $\mathrm{Y}$ indicates the prediction of images that is given as input as batches. We can specify the loss function which indicates how good the model's prediction is for the batch. Our target is to keep loss function as low as possible through our system to maintain the efficiency of detection. In this paper, the loss function is the cross-entropy between the target and softmax activation function which was used for prediction for a batch of images. It finds the probability error within the classes we make. It shows some problems for our detection system as the system can classify between revolver, machine gun and shot gun. It arises problems too. There are some features matched with other classes. While calculating loss function, our system shows high rate of loss function rate and sometimes totally misleading information while classifying gun types. But, in this paper it is not big issue as our purpose is only to detect gun. No matter what kind of gun a image contains. This occurs only while in bad quality images of gun but it can predict gun type perfectly for a batch of good quality images where cross-entropy is lower.

\subsubsection{Training Model}

We defined our loss function so far. In the part of the model we describe the steps how the system is trained using the tools we made. We optimize our error function using gradient optimizer and then run the training steps.

Gradient descent optimizer is a useful function that reduces error function using a learning rate. In this model, gradient descent optimizer uses gradient descent algorithm. At the time of feeding data to the models it is always considered to lower the learning rate to slow the process for gaining better accuracy. We set the learning rate to 0.003 in Gradient Descent Optimizer. This function takes the error function and computes the partial derivative of the error function relative all the weights and biases. We repeat this for upcoming batches of training images and reduce the cross-entropy.

Returned operation from gradient descent optimizer function updates the parameter of training. Training has to be run repeatedly with the increment of 50 images in each batch than previous batch. We followed the order of batches of images as 50, 100, 150, 200, 250, 300, 350,400 , and 450 . With every batch of training, it replaces the placeholders we created at 
software regression model. After this we evaluated the model and found the inefficiency in our model.

\subsection{Build Multilayer Convolutional Model}

To build multilayer convolutional network from the model we have created so far, we need to follow some steps like:

1. Weight Initialization

2. Convolution \& Pooling

3. First Convolutional Layer

4. Second Convolutional Layer

5. Densely Connected Layer

6. Dropout

7. Readout

8. Train and evaluate.

Weight initialization is important as we work with a lot of weights and biases in the models. We initialize the weights with a small amount of noise for symmetry breaking and to avoid 0 gradients. The value of weights with small amount of noise we use is 0.1 . Moreover, we use ReLu in our model. Therefore, to avoid "dead neurons", we use slightly positive initial bias. We initialize the bias with slightly positive values of 0.1 . These initialization processes is done within a function.

Convolution and pooling operation executes the task of handling boundaries. We keep convolutions of the stride of 1 and are zero padded to maintain the output as like input. We use pooling over a $2 \times 2$ blocks in our input images.

Stride: number of pixels to slide our filter matrix over the input matrix

Zero padding: padding the input matrix with zeroes.

In the datasets, we have images of $150 \times 100$. We divide the every image with $2 \times 2$ block with stride of 1 . As we use max pooling we take maximum value of the block and keep in the matrix. It helps to take the most weighted value so that we can take best value of predict the shape more efficiently. 
First convolutional layer computes 600 features for each $5 \times 5$ patch. For this layer weight variable will have a shape of 5, 5, 1, 600 in the parameter of making weight variable. In the shape the first two is patch size, then 1 is input channels and 600 is output channels. We kept limitation of 600 features as computation is always costly in terms of hardware, complexity and time. To execute the layer we need to reshape $\mathrm{X}$ with $150 \times 100$ and last parameter to reshape needs number of channels of color and in our case it is 1 as we are working on grayscale image. Then we use ReLu for de-noising.

An additional term, ReLU [2] is used in neural network operation. It is an element wise operation and it is used pixel per pixel. ReLU [2] replaces all the negative pixel values by zeros so in that way it would become non-linear. There is also various nonlinear functions like sigmoid or tanh instead of ReLU [2], but ReLu [2] has outdone all the other methods in terms of de-noising. For an input $150 \times 100$ pixel ReLU Scan through the full pixels and denoises to the edges. At the end of this layer, we use max pooling $(2 \times 2$ block $)$ over the images makes the size of original image to $75 \times 50$.

Second Convolutional layer does the same thing as first convolutional layer. Still it has little bit of in terms of taking parameters to construct weight. Weight takes 5, 5, 600,1200 as shape in the parameters of making variable. As mentioned above fist two element of parameters are patch number and here, input channels $=600$, output channels $=1200$. Then, again we run ReLu and pooling like first convolutional layer. Image size becomes half than before after running max pooling again $(37 \times 25)$.

Fully connected layer mainly gives output of probabilities of the classes we have in our dataset. After pooling operation, this layer is executed with the image size of $37 \times 25$. We add fully connected layer with 2048 neurons to process on the whole image. Weight of fully connected layer takes shape as 37, 25, 1200, 2048 in the parameter of making variable and bias variable takes shape as 2048 in the parameter of making variable. Then we reshape images from the pooling layer to batch of vectors and multiply by weight matrix and add a bias. We apply this whole calculation as parameter to ReLu [2]. Before getting the output as probability we apply dropout [18] to minimize overfitting. 
When the data from the fully connected layer is fetched, and showed in the accuracy graph there is always some exponential difference when output data is driven away from the given input test and there creates a large gap called overfitting in the accuracy graph of the data. To reduce the difference, a function dropout [18] is used. It flattens the images of the output value along size with input one resulting in much cleaner and accurate output. To implement dropout [18], we create a placeholder for probability on which the neuron's output is kept. We use the returned operation of ReLu from second fully connected layer and placeholder as parameters of dropout.

The last layer of this model is called readout layer. We make another variable of weight in this layer with the shape as 2048, 6 in the parameter. As mentioned above, we use 2048 to scan through the entire image and 6 because we have 6 classes. Again, a bias variable of taking shape 6 as parameter because of number of class we have in dataset. Then the retuned value of dropout function is multiplied with the weight variable created in readout layer and sums up with bias matrix and gives the prediction of multiple convolutional networks.

The last process we execute in our model is training and evaluating the model according to multiple convolutional networks. This process is as identical as we did in our model before to train simple software regression model. But there are few changes here than before:

- We use ADAM optimizer [19] in this part which is more sophisticated than Gradient descent optimizer.

- We keep a variable in parameters training to control dropout.

- We keep logging of every $50^{\text {th }}$ repeated task of training to keep information of result. 


\section{CHAPTER 4}

\section{Experimental Setup and Result analysis}

The system we have used was implemented using ImageNet datasets which contains 394 images of blood samples, 396 images of knife, 426 images of machinegun, 482 images of revolver, 406 images of shotgun and 302 images of gun in hand.

Table 1: Dataset information

\begin{tabular}{|c|c|c|}
\hline Testing Data & Training Data & Blood \\
\hline 400 & 394 & Knife \\
\hline 400 & 396 & Machinegun \\
\hline 400 & 426 & Revolver \\
\hline 400 & 482 & Shotgun \\
\hline 400 & 406 & Gun in hand \\
\hline 400 & 302 & \\
\hline
\end{tabular}

We gathered this dataset for our system's purpose where we made necessary changes such as cropping the raw image using the bounding box parameter that was given, then we divided each firearm and their respective models into different folders (the labels for both training and testing images were given). After that the implementation of algorithm is done, here we have used TensorFlow, which is an interface for training machine learning algorithms, and also executing such algorithms. It is an open source software library for numerical computation using data flow graphs. Nodes in the graph represent mathematical operations, while the graph edges represent the multidimensional data arrays (tensors) communicated between them. We have used Datasets of guns, knives, machineguns, revolvers and shotguns to detect whether there is a Threatening scene is going to happen or not.

So at a glance, the steps we followed are:

Step 1: Setting up Tensorflow.

Step 2: Creating a set of training images.

Step 3: Run the virtual machine.

Step 4: Link the images virtually. 
Step 5: Update the code.

Step 6: Compile the code.

Step 7: Create bottleneck files.

Step 8: Calculate train accuracy.

Step 9: Calculate validation accuracy.

Step 10: Calculate cross entropy.

Step 11: use a test sample to see the result.

\subsection{Setting up Tensorflow}

TensorFlow is an interface for training machine-learning algorithms, and performing such algorithms. It is an open source software library for numerical computation using data flow graphs. A computation done using TensorFlow can be performed on a wide variety of systems, ranging from mobile devices up to large-scale distributed systems. The system can be used to express a wide variety of algorithms for deep neural network models, and it has been used for conducting research and for deploying machine learning systems. Tensorflow can be set up by two process, for GPU support or CPU support. TensorFlow programs typically run significantly faster on a GPU than on a CPU. Unfortunately, as we don't have a high level GPU, we run tensorflow on a CPU based system.

\subsection{Creating a set of training dataset}

The dataset used is obtained from the Image net dataset. The dataset has been generated by supplying pictures from search engines like Google, Bing, etc. where noise free pictures are given out. Normal data could have been used but those would have had high level of noise margins which we have avoided for our training purposes in our convolutional models. For training to work well, we need to gather at least a hundred photos of each kind of object we want to recognize. In the whole of the dataset, 394 bloods sample, 302 gun in hand sample, 396 knife samples, 426 machinegun samples, 482 revolver samples, 406 shotgun samples. 


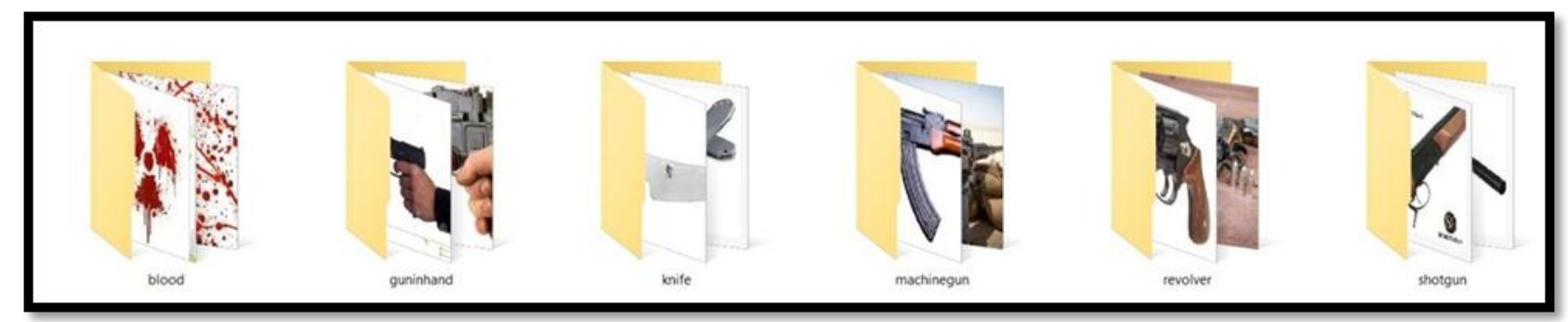

Fig 4.1: Image dataset
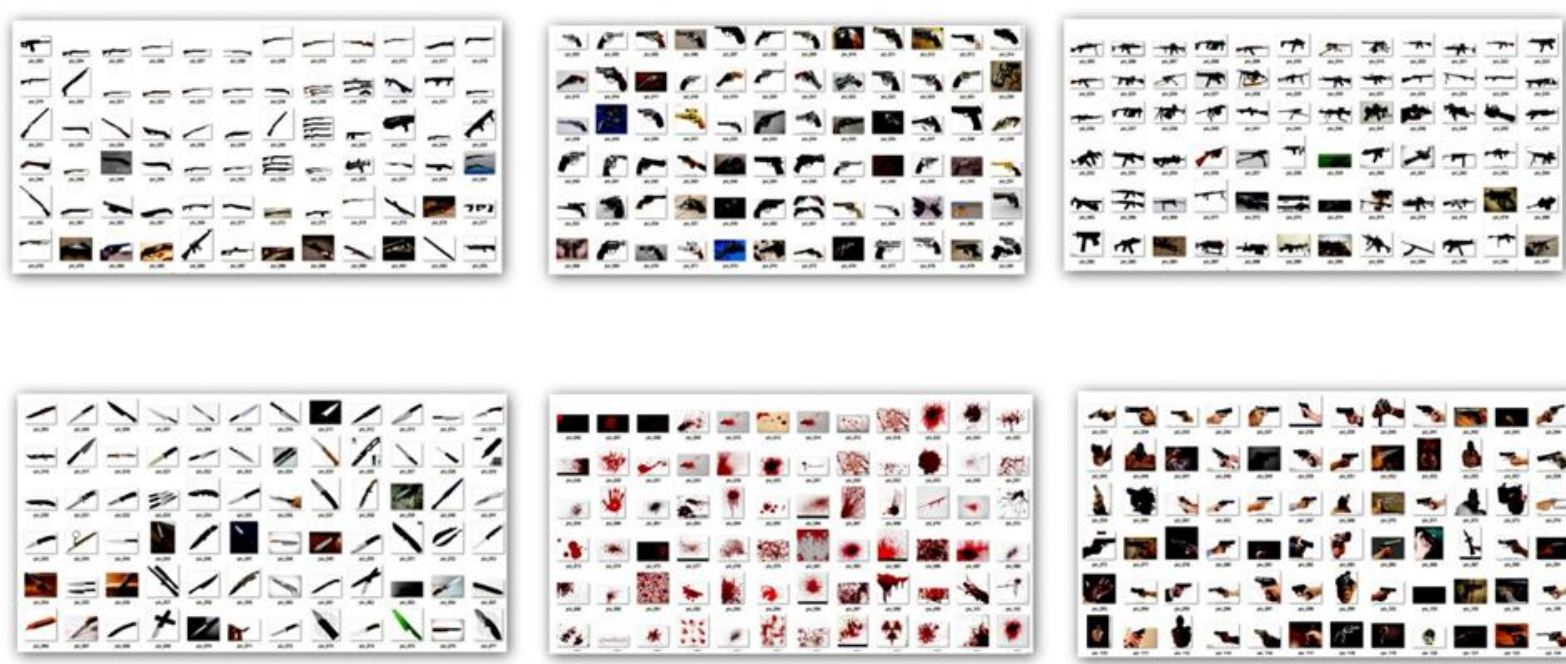

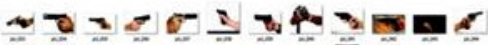

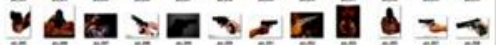

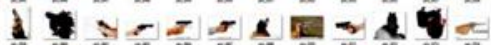

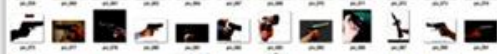

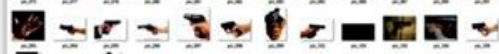

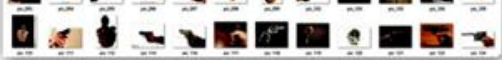

Fig 4.2: sample images

\subsection{Creating bottleneck files}

The first phase analyzes all the images on disk and calculates the bottleneck values for each of them. 'Bottleneck' is an informal term we often use for the layer just before the final output layer that actually does the classification, and this process is shown in Fig 4.3. This layer has been trained to output a set of values that's good enough for the classifier to use to distinguish between all the classes it's been asked to recognize. That means it has to be a meaningful and compact summary of the images, since it has to contain enough information for the classifier to make a good choice in a very small set of values. 


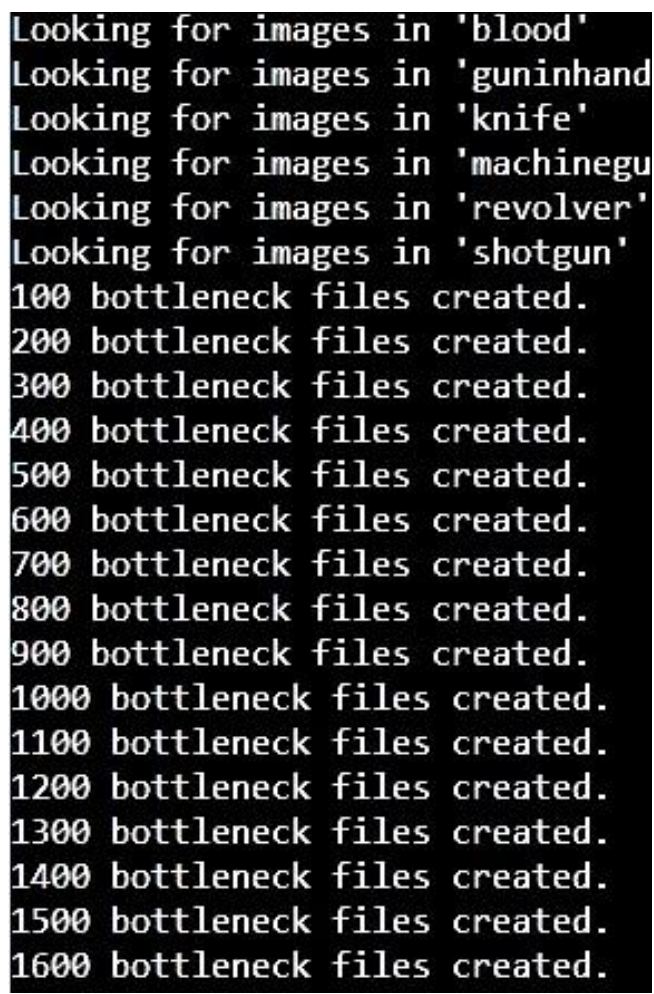

Fig 4.3: Creating bottleneck files

\subsection{Training}

Once the bottlenecks are complete, the actual training of the top layer of the network begins. In that time a series of step outputs, each one showing training accuracy, validation accuracy, and the cross entropy. The training accuracy shows what percent of the images used in the current training batch were labeled with the correct class. The validation accuracy is the precision on a randomly-selected group of images from a different set. Cross entropy is a loss function which gives a glimpse into how well the learning process is progressing. The key difference is that the training accuracy is based on images that the network has been able to learn from so the network can over fit to the noise in the training data. A true measure of the performance of the network is to measure its performance on a data set not contained in the training data -- this is measured by the validation accuracy. If the train accuracy is high but the validation accuracy remains low that means the network is over fitting and memorizing particular features in the training images that aren't helpful more generally. The training's objective is to make the loss as small as possible. 


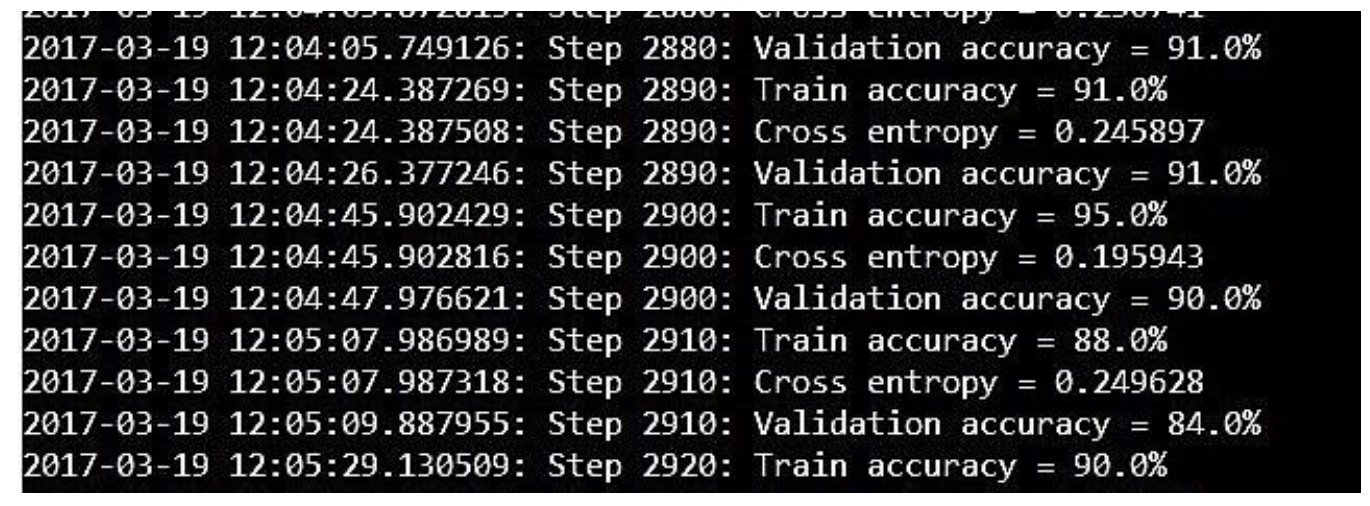

Fig 4.4: Training

In Fig 4.4, we can see that a screenshot of this script running 4,000 training steps. Each step chooses ten images at random from the training set. Finds their bottlenecks from the cache, and feeds them into the final layer to get predictions. Those predictions are then compared against the actual labels to update the final layer's weights through the back-propagation process. As the process continues you should see the reported accuracy improve, and after all the steps are done, a final test accuracy evaluation is run on a set of images kept separate from the training and validation pictures. This test evaluation is the best estimate of how the trained model will perform on the classification task. There will be an accuracy value of between $90 \%$ and $95 \%$, though the exact value will vary from run to run since there's randomness in the training process.

\subsection{Distortions}

A common way of improving the results of image training is by deforming, cropping, or brightening the training inputs in random ways. We enable these distortions by passing random crop,--random scale and random brightness to the script. These are all percentage values that control how much of each of the distortions is applied to each image. It's reasonable to start with values of 5 or 10 for each of them and then experiment to see which of them help. flip_left_right will randomly mirror half of the images horizontally, which makes sense as long as those inversions are likely to happen in the application. 


\subsection{Accuracy Measurement}

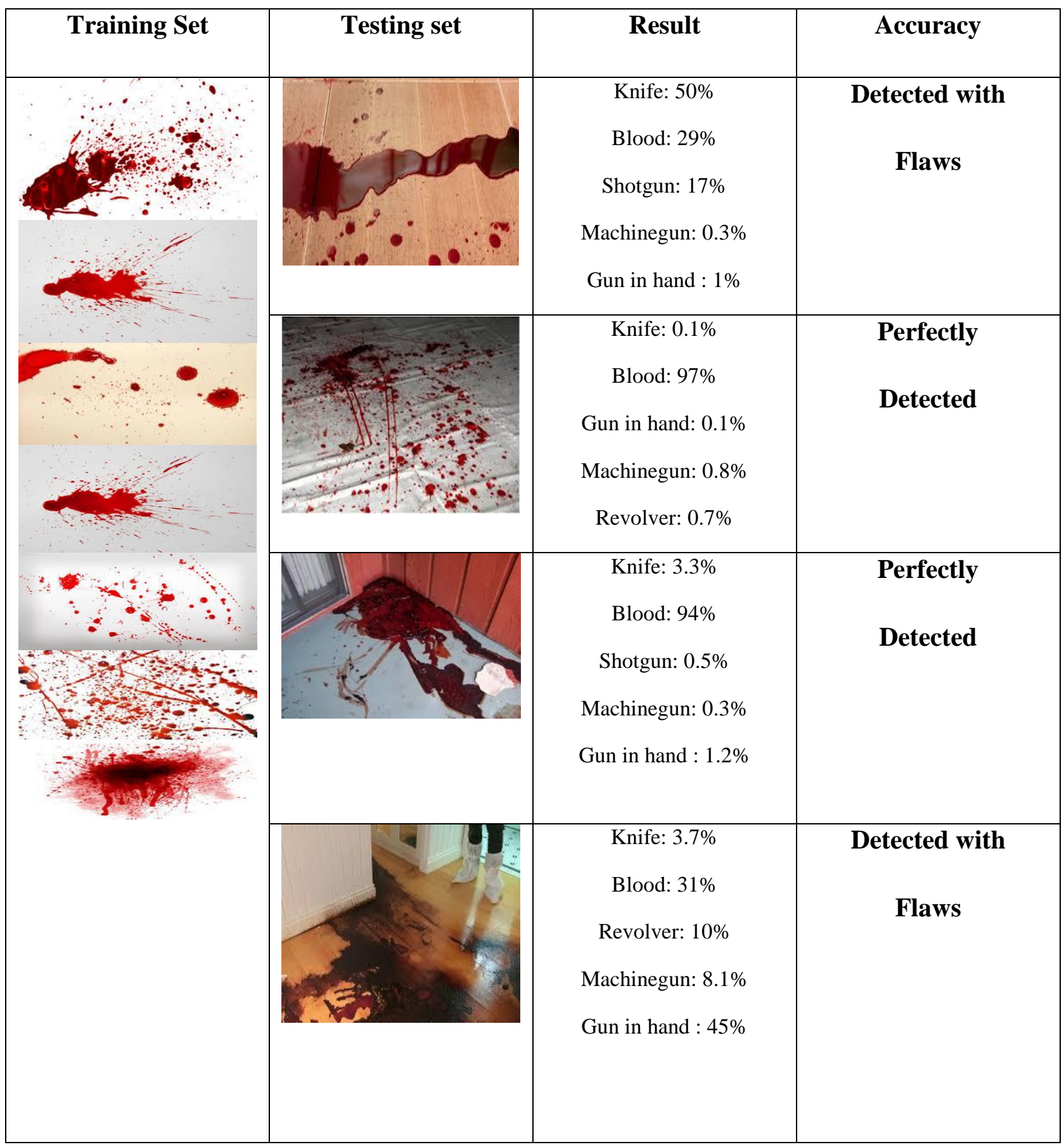

Fig 4.5: Blood Sample Testing 1

From this Fig 4.5, we can see that in most cases it successfully detects blood. In some cases, it gives fault. In real life scenario, the color, depth, anything can matter in detecting blood. So some mistakes will occur, as it is not fully prepared for any scenario. 


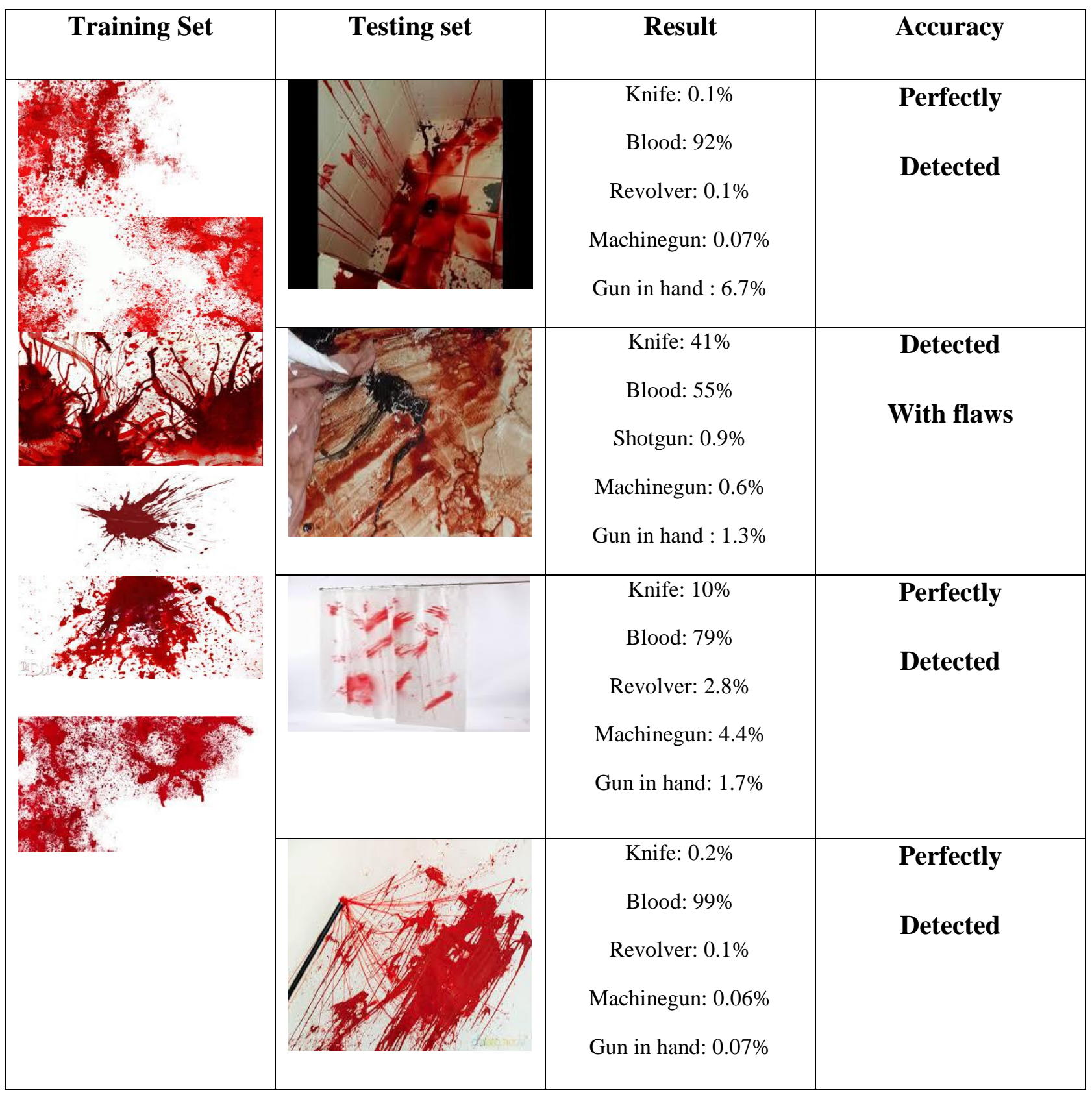

Fig 4.6: Blood Sample Testing 2

From this Fig 4.6, we can see that in most cases it successfully detects blood. In some cases, it gives fault. In real life scenario, the color, depth, anything can matter in detecting blood. So some mistakes will occur, as it is not fully prepared for any scenario.

From this Fig 4.7, we can see that in most cases it successfully detects Revolver. In some cases, it gives fault. In real life scenario, machinegun, shotgun and revolver has many similarities in many cases according to models and size, so it is pretty much easy for the classifier to confuse among them, so some error occurs. 


\begin{tabular}{|l|c|c|c|}
\hline Training Set & Result & Accuracy \\
\hline & Knife: $0.9 \%$ & Detected \\
\hline & Revolver: 5\% & Shotgun: $13 \%$ & Machinegun: $47 \%$ \\
\hline
\end{tabular}

Fig 4.7: Revolver Sample Testing 1

From this Fig 4.8, we can see that in most cases it successfully detects Revolver. In some cases, it gives fault. In real life scenario, machinegun, shotgun and revolver have many similarities in many cases according to models and size, so it is pretty much easy for the classifier to confuse among them, so some error occurs. 


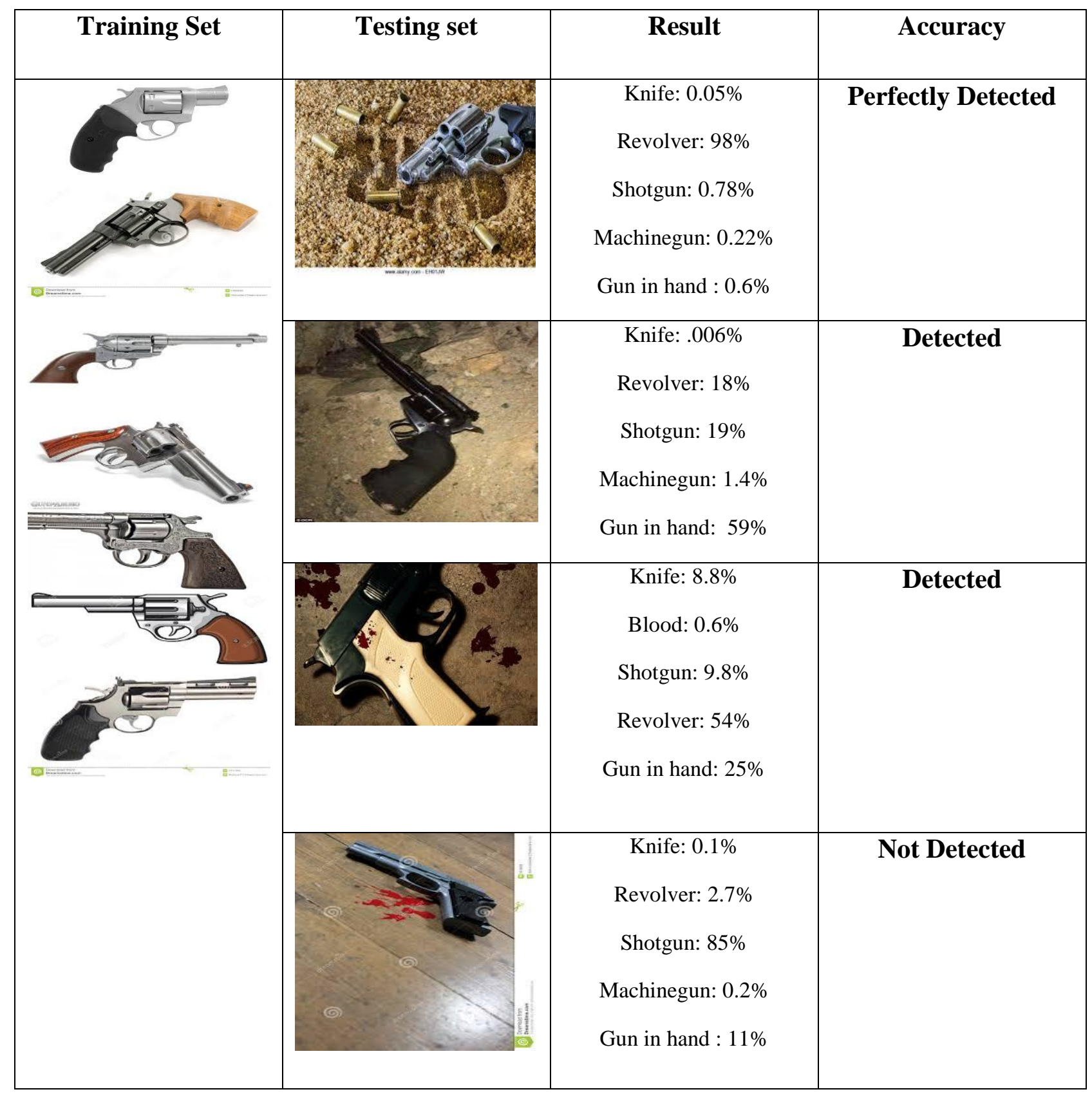

Fig 4.8: Revolver Sample Testing 2

From this Fig 4.9, we can see that in most cases it successfully detects Knife. In some cases, it gives fault. In real life scenario, the color, size, length and width anything can matter in detecting knife. So some mistakes will occur, as it is not fully prepared for any scenario. 


\begin{tabular}{|l|c|c|c|}
\hline Training Set & Result & Accuracy \\
\hline & Knife: 98\% & Perfectly Detected \\
\hline & Blood: 0.6\% & Shotgun: 0.2\% & \\
\hline & Machinegun: 0.1\% & Perfectly Detected \\
\hline & Revolver: 0.002\% & Knife: 90\% \\
\hline
\end{tabular}

Fig 4.9: Knife Sample Testing 1

From this Fig 4.10, we can see that in most cases it successfully detects Knife. In some cases, it gives fault. In real life scenario, the color, size, length and width anything can matter in detecting knife. So some mistakes will occur, as it is not fully prepared for any scenario. 


\begin{tabular}{|l|c|c|c|}
\hline Training Set & Result & Accuracy \\
\hline & Knife: $61 \%$ & Perfectly detected \\
\hline & Blood: $31 \%$ & Shotgun: $1.4 \%$ & \\
\hline & Machinegun: $5.5 \%$ & Rerting set & Revolver: $0.38 \%$ \\
\hline
\end{tabular}

Fig 4.10: Knife Sample Testing 1

From this Fig 4.11, we can see that in most cases it successfully detects machinegun. In some cases, it gives fault. In real life scenario, machinegun, shotgun and revolver have many similarities in many cases according to models and size, so it is pretty much easy for the classifier to confuse among them, so some error occurs. 


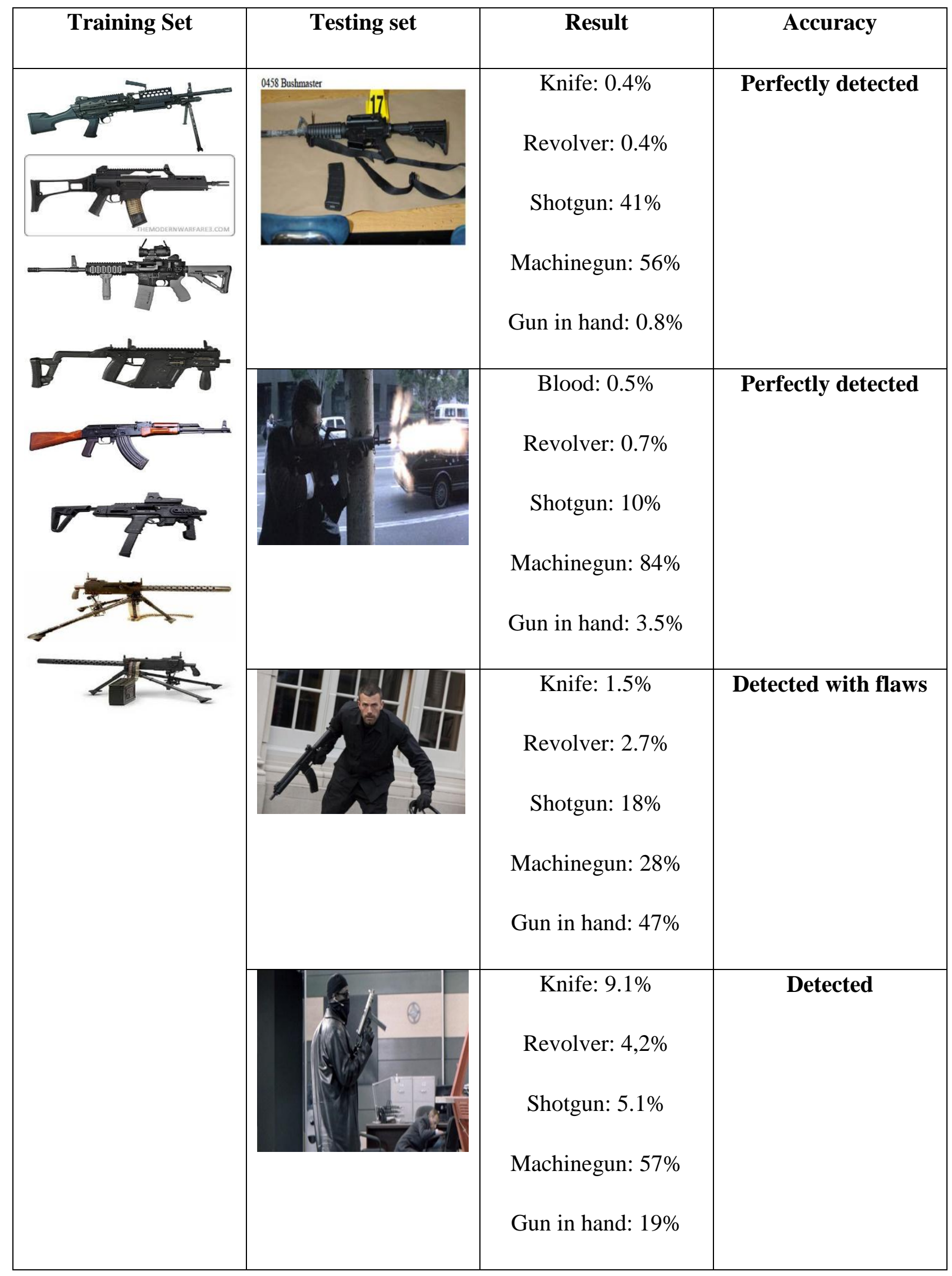

Fig 4.11: Machinegun Sample Testing 


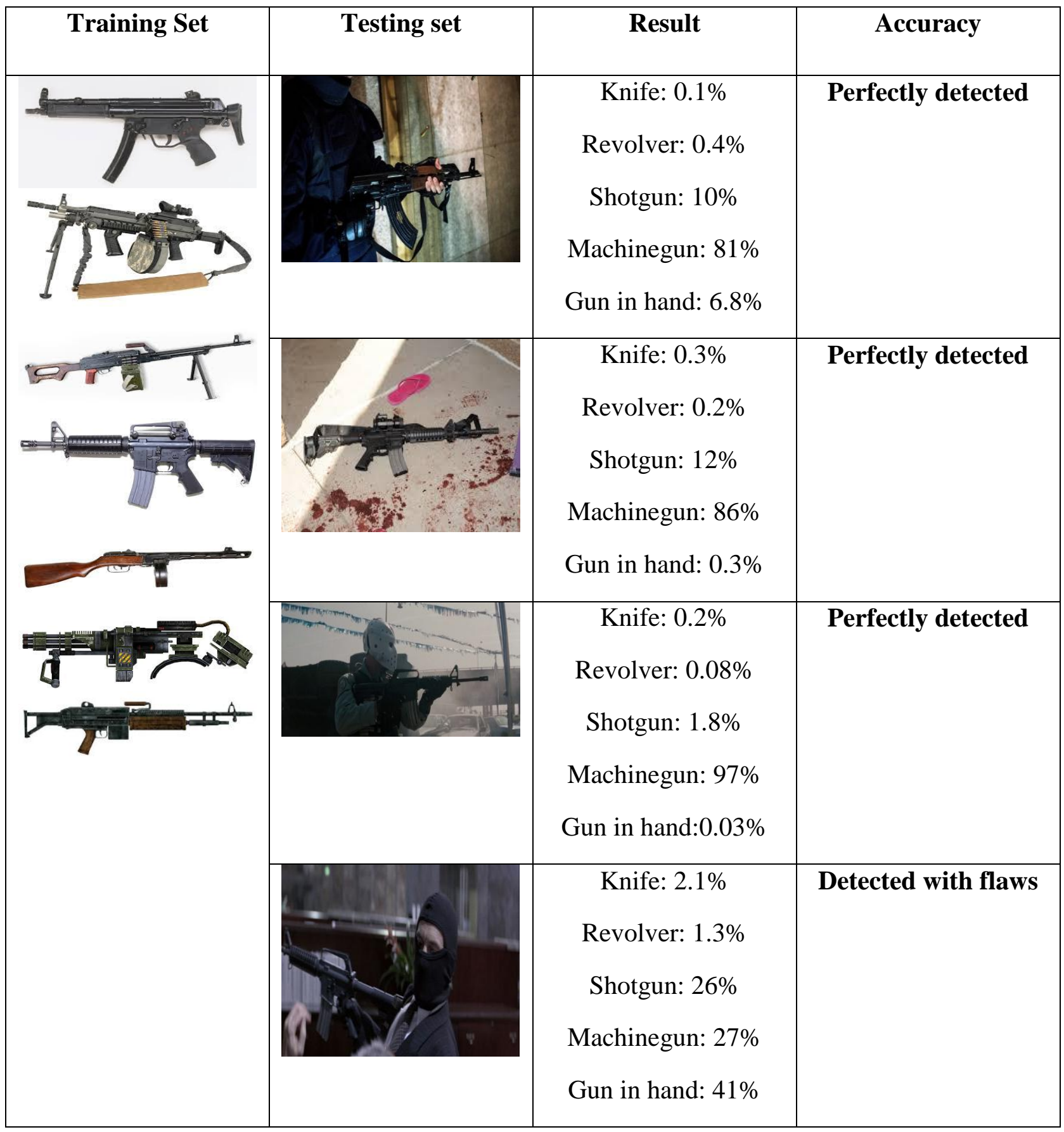

Fig 4.12: Machinegun Sample Testing 2

From this Fig 4.12, we can see that in most cases it successfully detects machinegun. In some cases, it gives fault. In real life scenario, machinegun, shotgun and revolver has many similarities in many cases according to models and size, so it is pretty much easy for the classifier to confuse among them, so some error occurs. 


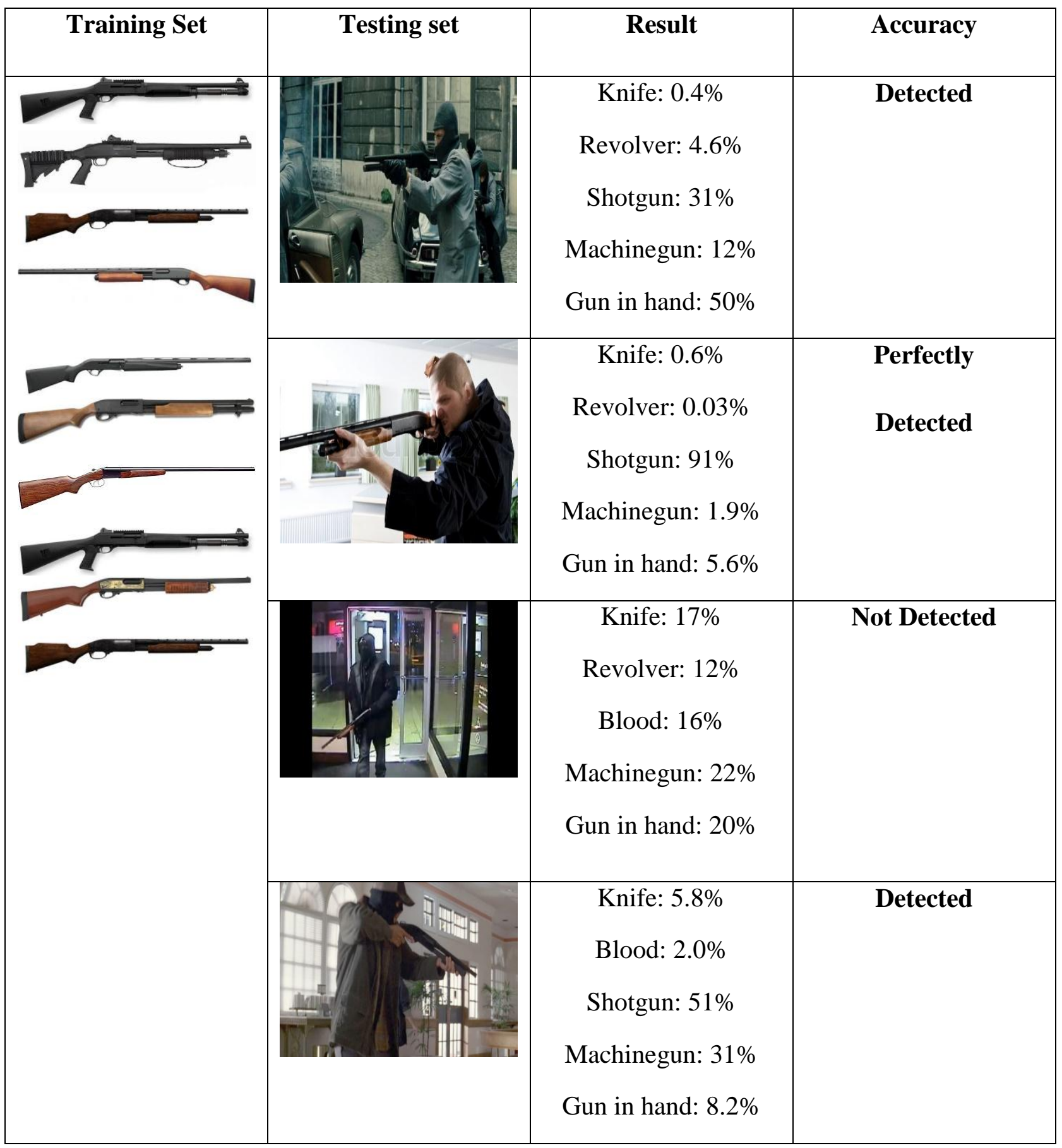

Fig 4.13: Shotgun Sample Testing 1

From this Fig 4.13, we can see that in most cases it successfully detects shotgun. In some cases, it gives fault. In real life scenario, machinegun, shotgun and revolver has many similarities in many cases according to models and size, so it's pretty much easy for the classifier to confuse among them, so some error occurs. 


\begin{tabular}{|l|l|l|}
\hline Training Set & Result & Accuracy \\
\hline & Knife: $1.5 \%$ & Detected with flaws \\
\hline & Revolver: $3.0 \%$ & Shotgun: $6.7 \%$ \\
\hline
\end{tabular}

Fig 4.14: Shotgun Sample Testing 2

From this Fig 4.14, we can see that in most cases it can't successfully detect shotgun. In most cases, it gives fault. In real life scenario, machinegun, shotgun and revolver has many similarities in many cases according to models and size, so it's pretty much easy for the classifier to confuse among them, so some error occurs. 


\subsection{Result Analysis}

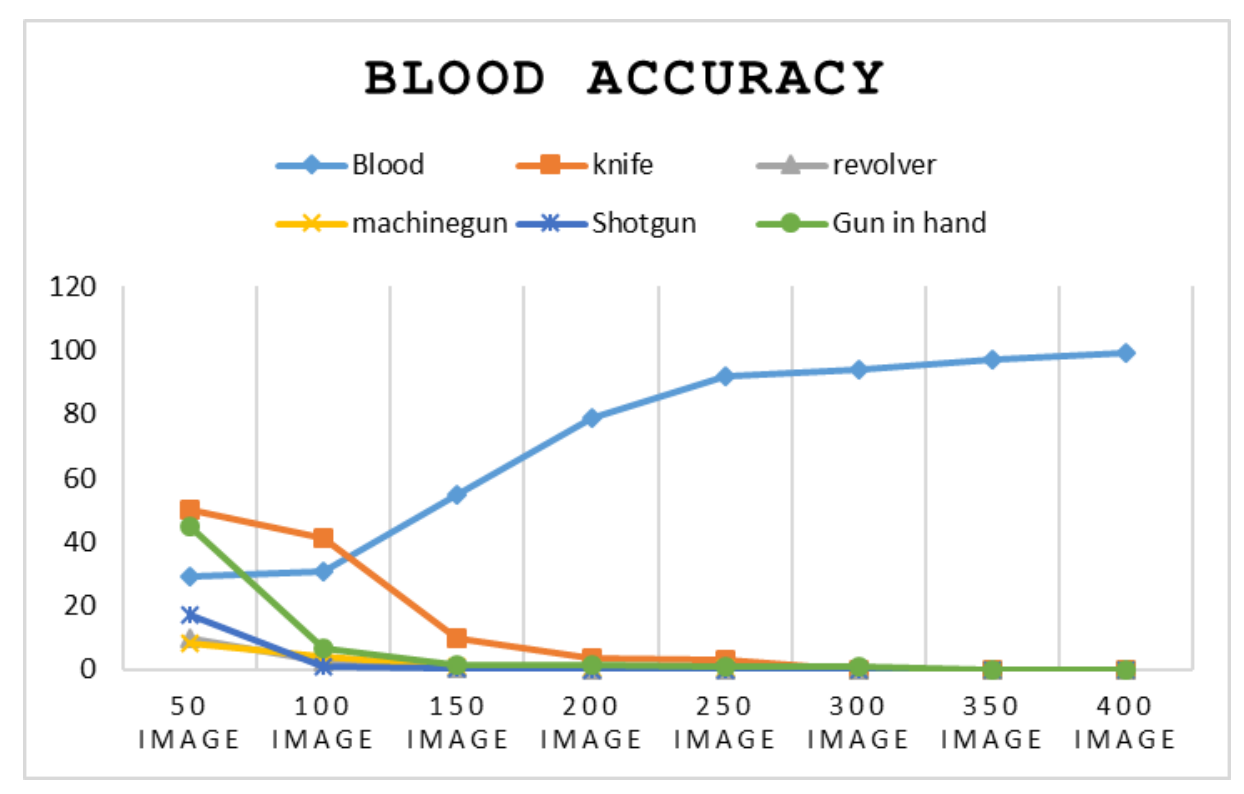

Fig 4.15: Blood accuracy graph

From the Fig 4.15 we can see that, as we have trained it for increasing number of blood samples, the accuracy percentage also increased from $29 \%$ to approximately $97 \%$ to $99 \%$.

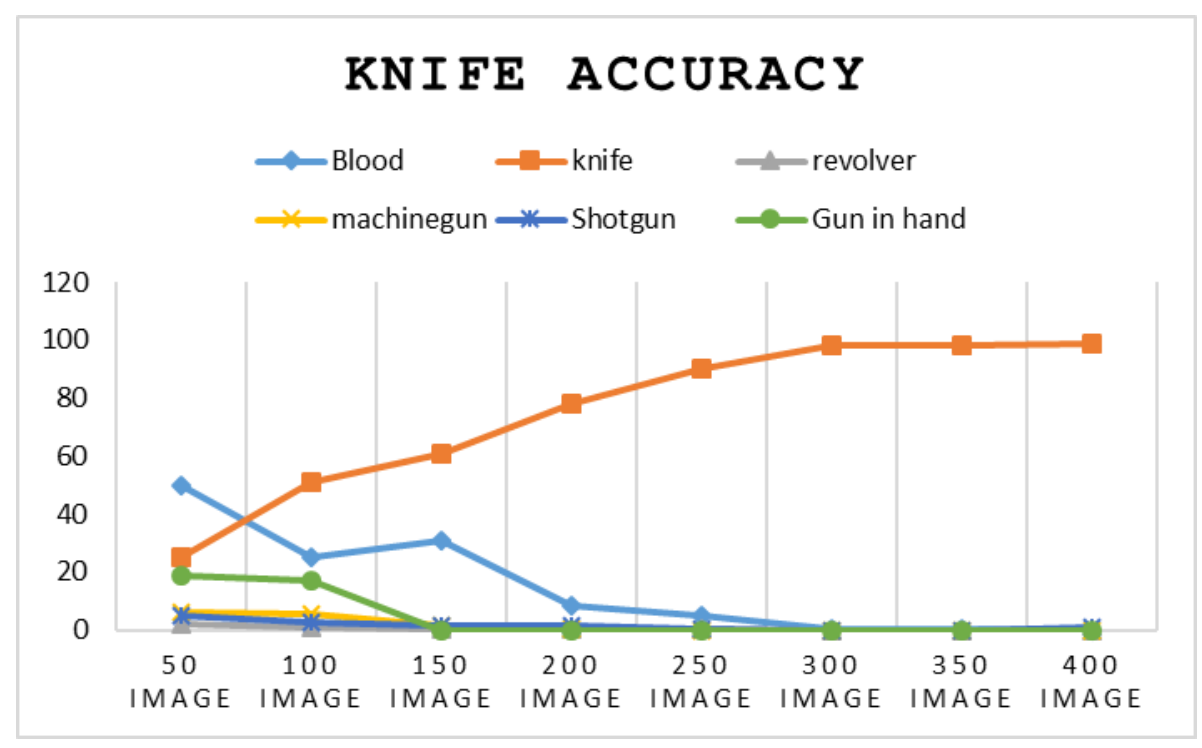

Fig 4.16: Knife accuracy graph 
From the Fig 4.16 we can see that, as we have trained it for increasing number of knife samples, the accuracy percentage also increased from $20 \%$ to approximately $98-99 \%$. But at same time in a real life scenario, the percentage can fluctuate.

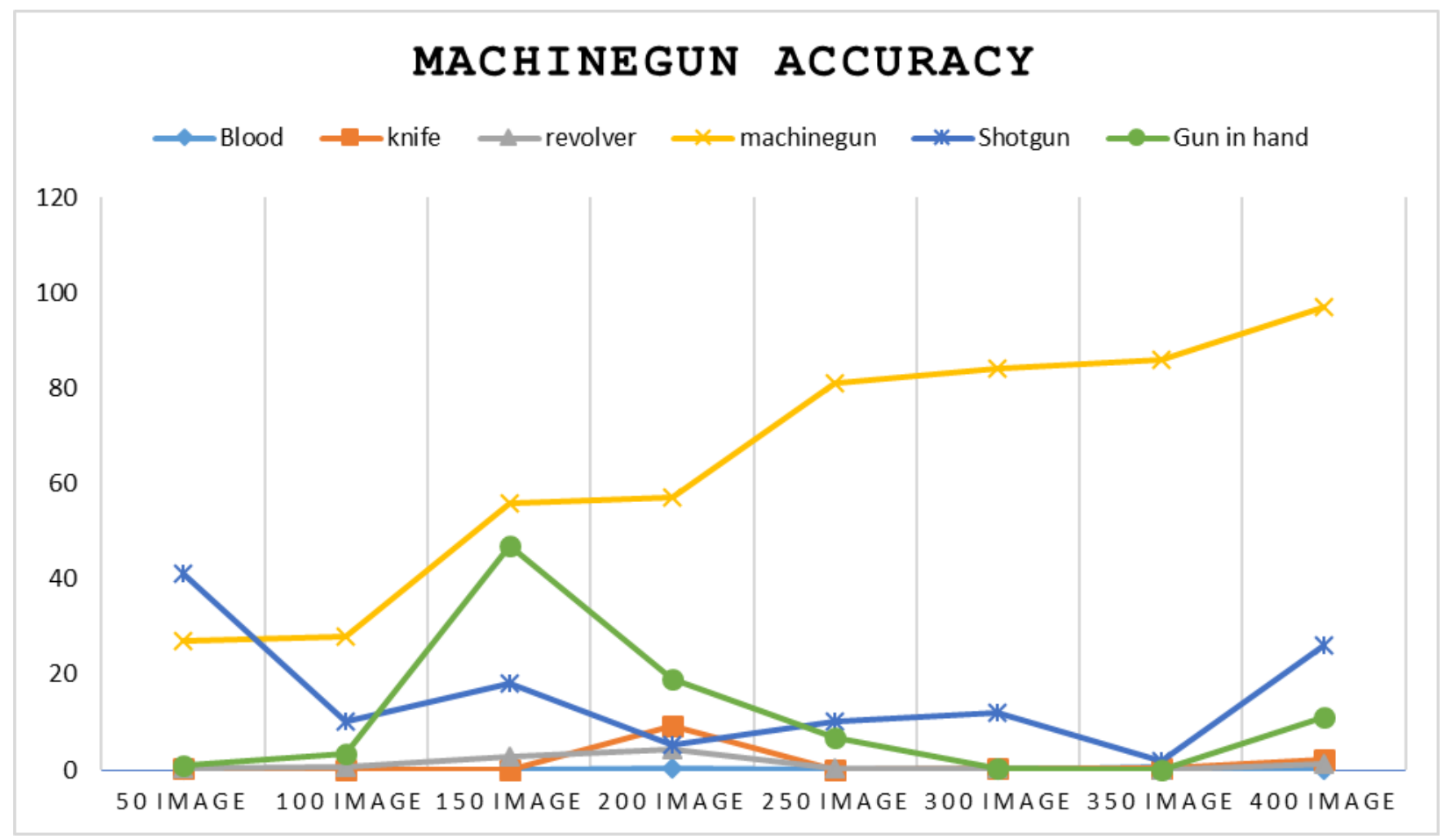

Fig 4.17: Machinegun accuracy graph

From the Fig 4.17 we can see that, as we have trained it for increasing number of shotgun samples, the accuracy percentage also increased from $25 \%$ to $85 \%$. But at same time in a real life scenario, the percentage can fluctuate.

From the figure 4.18 we can see that, as we have trained it for increasing number of revolver samples, the accuracy percentage also increased from $2.7 \%$ to $76 \%$. But at same time in a real life scenario, the percentage can fluctuate. 


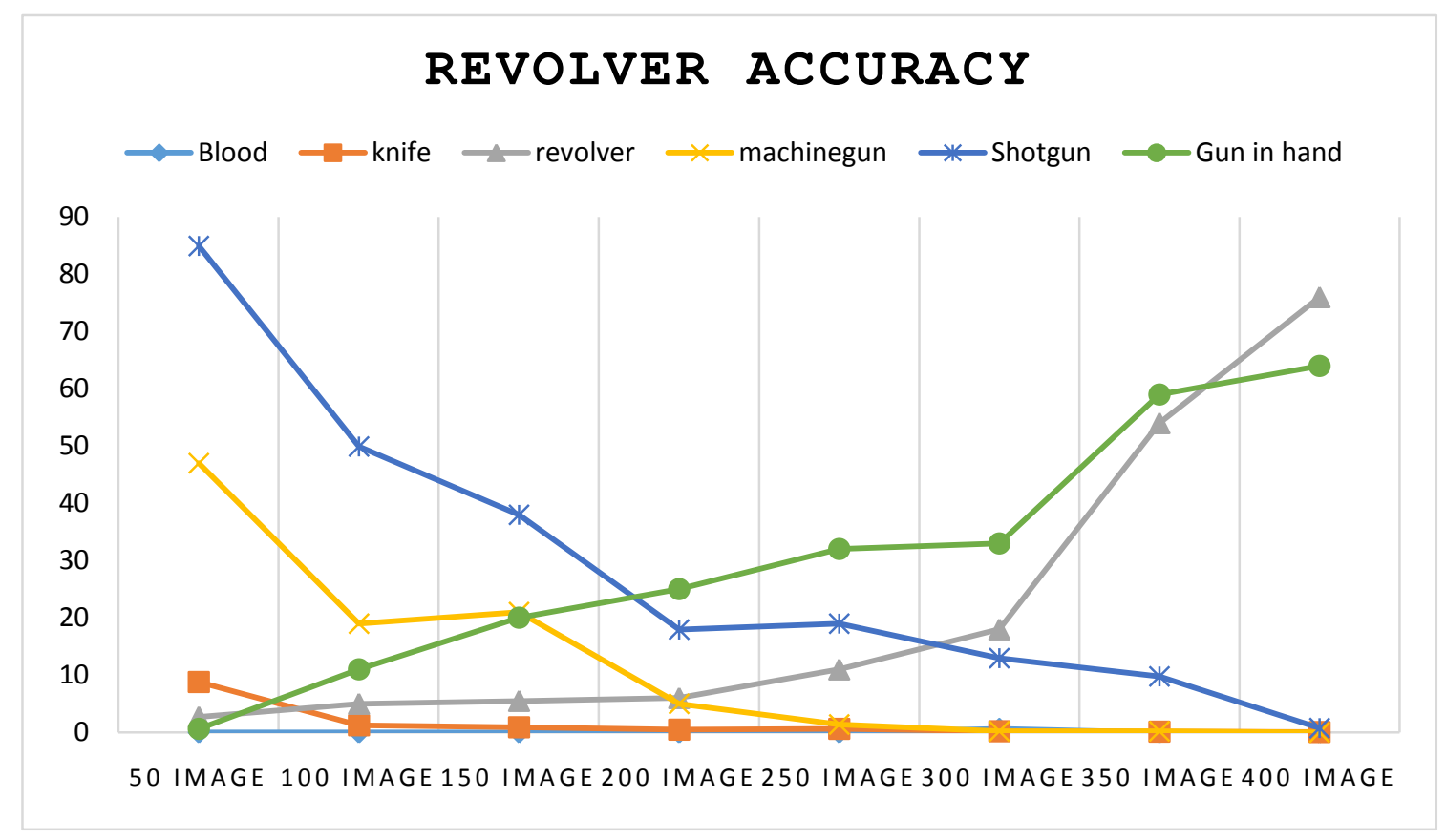

Fig 4.18: Revolver accuracy graph

From the figure 4.19, we summarized our final accuracy. Using our dataset for training the network for blood samples, we detected blood from various real life crime scenario. The problem here is, sometimes if anything occurs which's color is close to blood, the surveillance camera may misinterpret it as blood, so there are some areas we can develop for more accuracy. Also, in most cases, it perfectly detects blood, with additional objects. In some cases, where there is blood with knife, it can detect both. As we have trained it for mass amount and at the same time limited amount of blood in a scenario, it has gained an accuracy of almost 90 to 97 percent, which can also change because of real life scenario. At first, when we used 50-100 images as training sample, the accuracy lasted between 25 to 30 percent. But as we increased training data samples, after 200 images and 4000 iterations, the accuracy was between 90 to $99 \%$. 


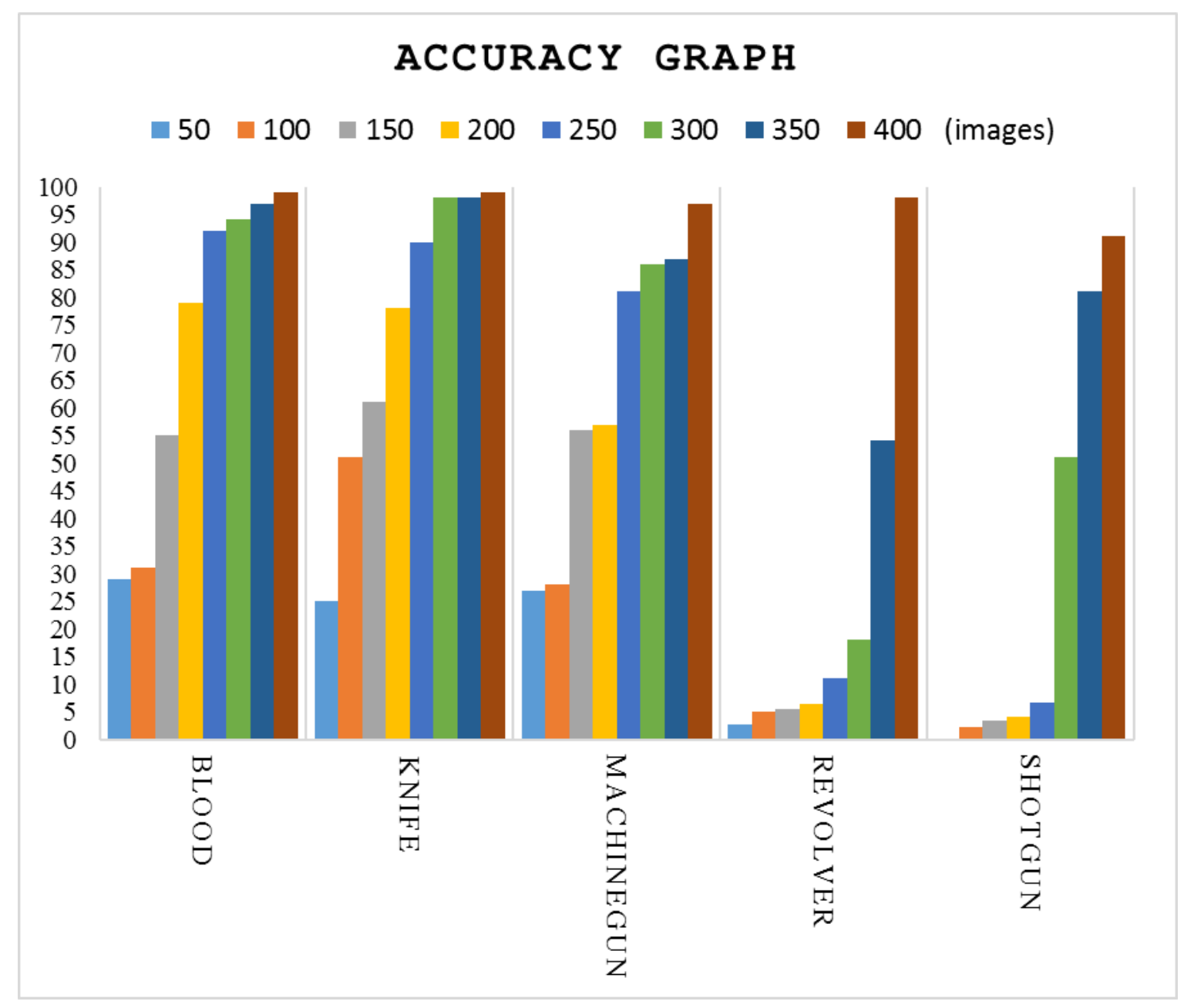

Fig 4.19: Final Accuracy graph

In case of training the network for knife samples, the graph shows good accuracy in detecting knife. The CNN successfully detects knife, because knifes shape is different from machinegun, revolver and shotguns. As we have trained it for different size, shape and angles in a scenario, it has gained an accuracy of almost 90 to 97 percent, which can also change because of real life scenario.

The main problem occurs in training revolver, machinegun and shotgun. When we first trained the $\mathrm{CNN}$ on machinegun, it was able to detect machinegun perfectly, because at that time we haven't trained the $\mathrm{CNN}$ on revolver and shotguns, but as soon as we trained the $\mathrm{CNN}$ model on both revolvers and shotguns, it becomes difficult for the $\mathrm{CNN}$ to differentiate. So for the first 50 to 200 sample images, as there are more than 100 models of different revolvers, shotguns and machineguns, it is not easy for the Model to correctly detect every class of firearms, as some models are pretty similar. 
Our main purpose was to detect every different class like knives, bloods, revolvers, shotguns and machineguns. Our model was able to perfectly detect blood and knives, but in case of firearms, it is not yet up to the mark. But the main purpose of guessing as if a crime has happened or not, can be guessed by detecting any type of firearms, whether it is a revolver, machinegun or shotgun. Also where there are multiple objects, like blood on knife, machineguns alongside a revolver - whatever the scenario is, it was able to detect multiple class from the same scenario. So we can say that the proposed model is accurate in most cases, with some exceptions which can be fixed by doing further research. 


\section{CHAPTER 05}

\section{CONCLUSIONS AND FUTURE WORKS}

\subsection{Concluding Remarks}

We can successfully detect blood, knife and gun on which we can predict the crime scene occurred or not. The wrong alert is reduced that makes us our model very efficient for this task compare to other models. To implement convolutional neural network, TensorFlow is the best platform we have found so far for this field and implanted in it. In this paper, we have 6 classes and for each of the class our system can detect the threatening objects and give result from an image. The results are given in percentage for each of the object we want to detect. Predicting crime scene by detecting threatening objects can have far reach impact on computer vision field. For our datasets, the test accuracy is $90.2 \%$ that is very competitive with the systems we have seen so far.

\subsection{Future Works}

- We work on a simple configured computer to implement the proposed model. In future, to fasten up the processing power it is needed to implement in high configured computer.

- The system has to be synced with surveillance camera and will try to implement very quickly in order to help law enforcement agencies.

- Detecting criminal using facial recognition along with this system will make the system more demanding. 


\section{REFERENCES}

[1] Abadi, Martín, et al. "TensorFlow: A system for large-scale machine learning." Proceedings of the 12th USENIX Symposium on Operating Systems Design and Implementation (OSDI). Savannah, Georgia, USA. 2016.

[2] "An Intuitive Explanation Of Convolutional Neural Networks". The data science blog, 2017. Web. 10 Feb. 2017. Available: https://ujjwalkarn.me/2016/08/11/intuitive-explanationconvnets

[3] "An open-source software library for Machine Intelligence". Web. 25 Dec. 2016. Available: https://www.tensorflow.org/

[4] Warden, Pete. "TensorFlow for Poets." Pete Warden's Blog. N.p., 18 Aug. 2016. Web. 04 Apr. 2017. Available: https://petewarden.com/2016/02/28/tensorflow-for-poets

[5]LiSourcell."LiSourcell/How_to_make_a_tensorflow_image_classifier_LIVE." GitHub.We b.04Mar.2017.Available:https://github.com/llSourcell/How_to_make_a_tensorflow_image_cl assifier_LIVE/blob/master/demonotes.ipynb

[6] Al-Rfou, Rami, et al. "Theano: A Python framework for fast computation of mathematical expressions." arXiv preprint arXiv: 1605.02688 (2016).

[7] "Learn TensorFlow and Deep Learning, without a Ph.D. | Google Cloud Big Data and Machine Learning Blog | Google Cloud Platform." Google Cloud Platform. Web. 10 Mar. 2017. Avaialable: https://cloud.google.com/blog/big-data/2017/01/learn-tensorflow-anddeep-learning-without-a-phd

[8] P. Sermanet and Y. Lecun, "Traffic sign recognition with multi-scale Convolutional Networks," The 2011 International Joint Conference on Neural Networks, 2011.

[9] K. He, X. Zhang, S. Ren, and J. Sun, “Deep Residual Learning for Image Recognition," 2016 IEEE Conference on Computer Vision and Pattern Recognition (CVPR), 2016K. He, X. Zhang, S. Ren, and J. Sun, "Deep Residual Learning for Image Recognition,” 2016 IEEE Conference on Computer Vision and Pattern Recognition (CVPR), 2016.

[10] Y. Xu, T. Mo, Q. Feng, P. Zhong, M. Lai, E. I. Chang, et al. Deep learning of feature representation with multiple instance learning for medical image analysis. In ICASSP, 2014.

[11] Erhan, Dumitru, Christian Szegedy, Alexander Toshev, and Dragomir Anguelov. "Scalable Object Detection Using Deep Neural Networks." 2014 IEEE Conference on Computer Vision and Pattern Recognition (2014).

[12] He, K., Gkioxari, G., Dollár, P., \& Girshick, R. (2017). Mask R-CNN. Cornell University Library. 
[13] D. Lowe. “Object recognition from local scale-invariant features”. In ICCV, 1999.

[14] N. Dalal and B. Triggs. "Histograms of oriented gradients for human detection". In CVPR, 2005.

[15] Ali, Khawlah Hussein, and Tianjiang Wang. "Learning Features for Action Recognition and Identity with Deep Belief Networks." 2014 International Conference on Audio, Language and Image Processing (2014).

[16] O'reilly, Dean, Nicholas Bowring, and Stuart Harmer. "Signal Processing Techniques for Concealed Weapon Detection by Use of Neural Networks." 2012 IEEE 27th Convention of Electrical and Electronics Engineers in Israel (2012).

[17] Grega, Michael, Andrzej Matiolanski, Piotr Guzik, and Mikolaj Leszczuk. "Automated Detection of Firearms and Knives in a CCTV Image." Sensors 16.1 (2016): 47

[18] Nitish Srivastava, Geoffrey Hinton, Alex Krizhevsky, Ilya Sutskever, and Ruslan Salakhutdinov. 2014. Dropout: a simple way to prevent neural networks from overfitting. $J$. Mach. Learn. Res. 15, 1 (January 2014), 1929-1958.

[19] Kingma, Diederik P., and Jimmy Ba. "Adam: A Method For Stochastic Optimization". 3rd International Conference for Learning Representations, San Diego, 2015 v1 (2014). 